\title{
The Activators of Type 2A Phosphatases (PP2A) Regulate Multiple Cellular Processes Via PP2A-Dependent and -Independent Mechanisms in Fusarium graminearum
}

\author{
Zunyong Liu, ${ }^{1} \mathrm{Na}$ Liu, ${ }^{1}$ Huixian Jiang, ${ }^{1}$ Leiyan Yan, ${ }^{2}$ Zhonghua Ma, ${ }^{1,3,+}$ and Yanni Yin ${ }^{1,+}$ \\ ${ }^{1}$ Institute of Biotechnology, Key Laboratory of Molecular Biology of Crop Pathogens and Insects, Zhejiang University, 866 \\ Yuhangtang Road, Hangzhou 310058, China; ${ }^{2}$ Ningbo Academy of Agricultural Sciences, Ningbo, 315040, China; and ${ }^{3}$ State \\ Key Laboratory of Rice Biology, Zhejiang University
}

Accepted 12 May 2018.

\begin{abstract}
The type 2A protein phosphatases (PP2As) are holoenzymes in all eukaryotes but their activators remain unknown in filamentous fungi. Fusarium graminearum contains three PP2As (FgPp2A, FgSit4, and FgPpg1), which play critical roles in fungal growth, development, and virulence. Here, we identified two PP2A activators (PTPAs), FgRrd1 and FgRrd2, and found that they control PP2A activity in a PP2A-specific manner. FgRrd1 interacts with FgPpg1, but FgRrd2 interacts with FgPp2A and very weakly with FgSit4. Furthermore, FgRrd2 activates FgPp2A via regulating FgPp2A methylation. Phenotypic assays showed that FgRrd1 and FgRrd2 regulate mycelial growth, conidiation, sexual development, and lipid droplet biogenesis. More importantly, both FgRrd1 and FgRrd2 interact with RNA polymerase II, subsequently modulating its enrichments at the promoters of mycotoxin biosynthesis genes, which is independent on PP2A. In addition, FgRrd2 modulates response to phenylpyrrole fungicide, via regulating the phosphorylation of kinase FgHog1 in the high-osmolarity glycerol pathway, and to caffeine, via modulating FgPp2A methylation. Taken together, results of this study indicate that FgRrd1 and FgRrd2 regulate multiple physiological processes via different regulatory mechanisms in F. graminearum, which provides a novel insight into understanding the biological functions of PTPAs in fungi.
\end{abstract}

Type 2A protein phosphatase 2A (PP2A) holoenzymes comprise a family of serine and threonine phosphatases and play important roles in cell-cycle regulation, cell morphology and development, and intracellular signal transduction, and other physiological processes (Westermarck and Hahn 2008). Heterotrimeric PP2A holoenzymes include a catalytic C subunit, a

${ }^{\dagger}$ Corresponding authors: Zhonghua Ma; E-mail: zhma@zju.edu.cn and Yanni Yin; E-mail: ynyin@zju.edu.cn

Funding: This research was supported by the National Natural Science Fund for Distinguished Young Scholar (31525020), National Science Foundation (31571945, 31401696), China Agriculture Research System (CARS-3-1-15), the Fundamental Research Funds for the Central Universities (2017FZA6014), Dabeinong Funds for Discipline Development and Talent Training in Zhejiang University, and China Postdoctoral Science Foundation (2017M620250).

*The $\boldsymbol{e}$-Xtra logo stands for "electronic extra" and indicates that five supplementary figures and one supplementary table are published online.

(c) 2018 The American Phytopathological Society structural A subunit, and a regulatory B subunit responsible for substrate specificity (Mumby 2007; Shi 2009; Xu et al. 2006). The catalytic activity of the PP2A holoenzymes is important for their functions and is controlled by a sophisticatedly coordinated network of several PP2A modulators (Sents et al. 2013; Stanevich et al. 2011). PP2A activators (PTPAs), as one class of the PP2A modulators, are highly conserved proteins from fungi to animal, but their functions vary significantly in different organisms (Cayla et al. 1994; Van Hoof et al. 1998). Human PTPA, encoded by a single gene PTPA, is involved in cell proliferation and survival and is regulated by the ubiquitous transcriptional factor Yin Yang 1 and the mitogen-activated protein kinase (MAPK) p53 (Guo et al. 2014; Janssens et al. 1999, 2000), whereas Saccharomyces cerevisiae PTPAs are encoded by two rapamycin-resistant deletion $(R R D)$ genes, RRD1 and RRD2 (Rempola et al. 2000). Both Rrd1 and Rrd2 are responsible for sensitivity to rapamycin and modulation of cell cycle during the $\mathrm{G} 1$ phase or $\mathrm{M}$ phase, dependent on the target of rapamycin (TOR) pathway (Leulliot et al. 2006; Van Hoof et al. 1998, 2000, 2001). Further study found that PTPA homologs in humans and budding yeasts regulate both the tyrosyl phosphatase activity and the Ser/Thr phosphatase activity of PP2A via isomerizing the 190 proline or stabilizing the protein fold of PP2A catalytic subunit in the presence of ATP/ $\mathrm{Mg}^{2+}$ (Fellner et al. 2003; Jordens et al. 2006). A recent study showed that human PTPA also can activate PP2A by reducing its phosphorylation at 307 tyrosine via upregulation of protein tyrosine phosphatase 1B (Luo et al. 2013). In Schizosaccharomyces pombe, PTPA homolog Ypa2 plays roles in regulating cell polarity and rounded morphology via interacting with $\mathrm{Cdc} 42$ regulators and the polarity factors Tea1, Tea4, and For3 (Bernal et al. 2012). In filamentous fungi, although the roles of PP2A subunits have been studied in several fungi, namely, Aspergillus nidulans, Ustilago maydis, Fusarium verticillioides, and Magnaporthe oryzae (Albataineh et al. 2014; Du et al. 2013; Egan et al. 2009; Shin et al. 2013; Zhong et al. 2014), the biological functions of PTPAs have not been characterized until now.

Fusarium graminearum is the major causal agent of Fusarium head blight (FHB), which is a devastating disease of cereal crops worldwide (Starkey et al. 2007). In addition to the yield loss caused by $F$. graminearum, the mycotoxins, such as deoxynivalenol (DON) and its derivatives in infested grains, arouse a serious threat to human and animal health (Desai and Mitchison 1997; McMullen et al. 1997; Pestka and Smolinski 2005). Our previous study characterized the biological functions of three $\mathrm{PP} 2 \mathrm{As}$ in F. graminearum. FgPp2A is essential for survival, 
FgSit4 and FgPpg1 play important roles in mycelial growth, virulence, lipid droplet biogenesis, and cell-wall integrity (CWI) (Yu et al. 2014). Therefore, we initiated this project to explore the molecular mechanism of PP2As regulated by PTPAs (FgRrd1 and FgRrd2) in F. graminearum, an emerging model for the assessment of fungal development. In this study, we found that FgRrd1 and FgRrd2 interact with specific FgPP2As and regulate their activity. Moreover, FgRrd 2 modulates FgPp2A activity via altering the methylation level of $\mathrm{FgPp} 2 \mathrm{~A}$, which is associated with the response to caffeine. Interestingly, FgRrd1 and FgRrd2 mutants showed significantly lower transcription of DON biosynthesis genes than the PP2A mutants. Further studies revealed that FgRrd1 and FgRrd2 interact with RNA polymerase II and modulate its enrichment at the promoters of DON biosynthesis genes, which is independent on PP2As. This study identified two different regulatory mechanisms of PTPAs in an important pathogenic fungus, which is helpful for the study on the biological functions of PTPAs in fungi.

\section{RESULTS}

Identification of PTPAs in F. graminearum.

In-silico analyses showed that the $F$. graminearum genome contains two PTPA orthologs, which were named FgRrd1 (FGSG_09229) and FgRrd2 (FGSG_01092), according to $S$. cerevisiae Rrd1 and Rrd2. FgRrd1 and FgRrd2 are predicted to encode a 446- and 434-amino acid protein, respectively. Phylogenic and domain analyses showed that FgRrd1 and FgRrd2 are homologous to PTPA orthologs from other fungal species and humans, and all PTPA orthologs comprise a conserved PTPA domain (Fig. 1A and B).

To characterize FgRRD1 and $F g R R D 2$, we first tested whether these genes are able to complement the defects of the yeast $R R D$ mutants. An expression vector pYES2 containing the fulllength $F g R R D 1$ cDNA was transformed into yeast RRDI mutant BY4741 $\Delta$ Rrd1. The mutant transformed with an empty pYES2 vector served as a negative control. As shown in Figure $1 \mathrm{C}$, sensitivity of yeast BY4741 $\Delta$ Rrd1 to rapamycin (targeting

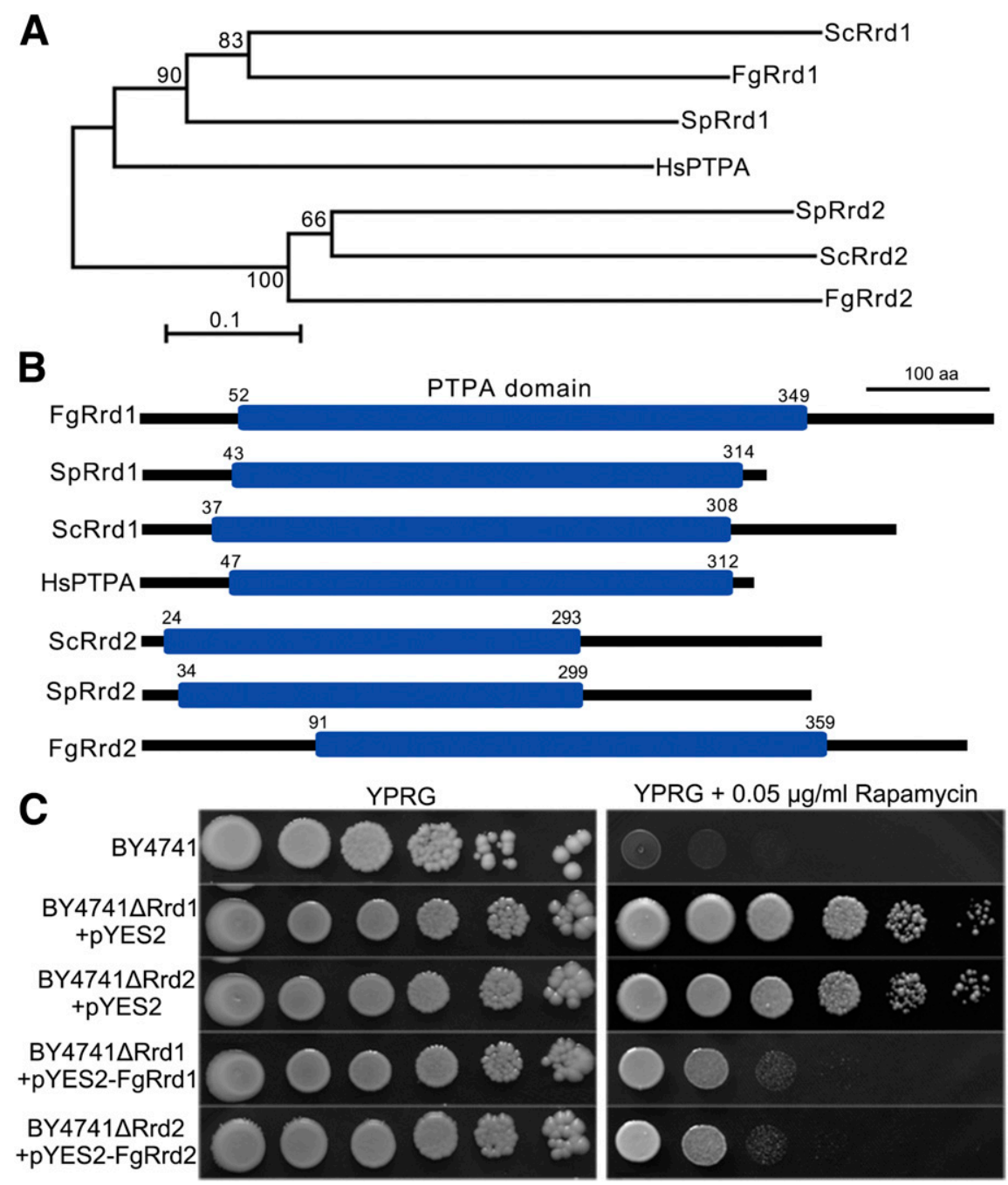

Fig. 1. FgRrd1 and FgRrd2 from Fusarium graminearum are counterparts of Saccharomyces cerevisiae Rrd1 and Rrd2, respectively. A, A phylogenetic tree generated using the neighbor-joining method, with Mega 5.0 software, based on deduced amino acid sequences of PP2A activator (PTPA) orthologs from F. graminearum (FgRrd1), Schizosaccharomyces pombe (SpRrd1, SPAC4F10.04; SpRrd2, SPAC1782.05), Saccharomyces cerevisiae (ScRrd1, YIL153W; ScRrd2, YPL152W), and Homo sapiens (HsPTPA, ENSG00000119383). The bootstrap values from 1,000 replications are indicated on the branches. B, PTPAs contain a conserved PTPA domain with the amino acid positions identified by the National Center for Biotechnology Information database. $\mathbf{C}$, $F$. graminearum FgRrd1 and FgRrd2 partially restored sensitivity of the yeast PTPA mutants BY4741 $\Delta$ Rrd1 and BY4741 $\Delta$ Rrd2, respectively, to rapamycin. Cells of the transformants containing pYES2, pYES2-FgRrd1, or pYES2-FgRrd2 were spotted onto YPRG medium (1\% yeast extract, 2\% bactopeptone, $2 \%$ galactose) supplemented with $0.05 \mu \mathrm{g}$ of rapamycin per milliliter. In addition, the wild-type BY4741 transformed with the empty pYES2 was used as a control. 
the TOR kinase) was restored by genetic complementation of the mutant with F. graminearum FgRRD1. Similarly, FgRRD2 could restore tolerance of the BY4741 $\Delta \mathrm{Rrd} 2$ mutant to rapamycin. These results indicate that $F$. graminearum $\mathrm{FgRrd} 1$ and FgRrd 2 and yeast Rrd1 and Rrd2 share a conserved function in response to rapamycin.

\section{FgRrd1 and FgRrd2 interact with specific FgPP2A.}

To further investigate the roles of FgRRD1 and FgRRD2 in $F$. graminearum, we generated gene-deletion mutants using a homology recombination strategy (Supplementary Fig. S1). The resulting hygromycin-resistant transformants of each gene were screened by polymerase chain reaction (PCR) with the primer pairs listed in Supplementary Table S1 and were further confirmed to be null mutants resulting from homologous recombination events at the corresponding gene locus, by Southern hybridization assays. Furthermore, to confirm that phenotypic changes observed in $\Delta \mathrm{FgRrd} 1$ or $\Delta \mathrm{FgRrd} 2$ were due to the deletion of the FgRRD1 or FgRRD2 gene, the mutant was complemented with a full-length wild-type $F g R R D 1$ or $F g R R D 2$ gene, respectively. However, we failed to obtain the FgRRD1 and FgRRD2 double-deletion mutant after screening 300 ectopic transformants, indicating deletion of both genes is lethal.
Previous studies have found that PTPA orthologs in S. cerevisiae regulate PP2A activity (Fellner et al. 2003; Jordens et al. 2006). We therefore explored the functions of FgRrd1 and FgRrd2 in activating PP2A in F. graminearum. Phosphatase activity assays showed that the PP2A activity was dramatically reduced in $\Delta \mathrm{FgRrd} 1$ and $\Delta \mathrm{FgRrd} 2$ compared with that in the wild type and complemented strains (Fig. 2A). Our previous study showed that $F$. graminearum contains three PP2As (FgPp2A, FgSit4, and FgPpg1) (Yu et al. 2014). To explore regulatory mechanism of PTPA on PP2A activity, we determined the interaction of FgRrd1 and FgRrd2 with the three FgPP2As, using coimmunoprecipitation (Co-IP) and yeast two-hybrid (Y2H) assays. As shown in Figure 2B, FgRrd1 interacted with FgPpg1, FgRrd2 interacted with FgPp2A and weakly with FgSit4 in the Co-IP assays. However, the Y2H assays showed that PTPAs did not interact with PP2A directly (Fig. 2C). In a previous study, we found that PP2A-associating protein FgTap42 interacted with three PP2As in F. graminearum (Yu et al. 2014). Thus, we further tested interaction of PTPA with FgTap42, by the Y2H assay. As shown in Figure 2C, FgTap42 interacted with both FgRrd1 and FgRrd2. In addition, the subcellular localization assay revealed that FgRrd1-GFP (green fluorescent protein), FgRrd2-GFP, FgTap42-GFP were all localized into cytosol and nucleus (Fig.
A

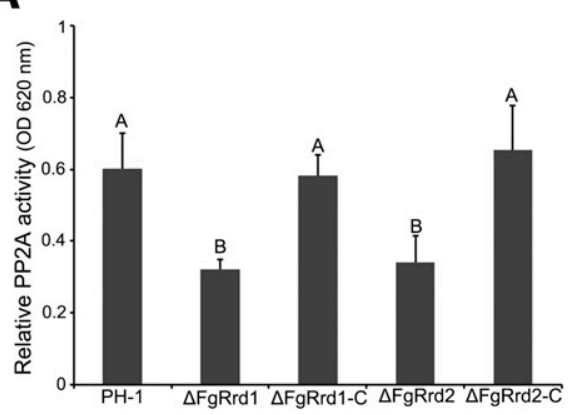

C

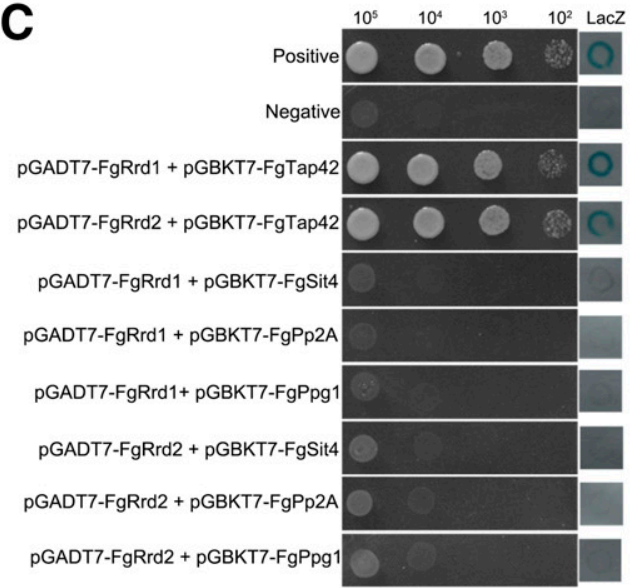

B
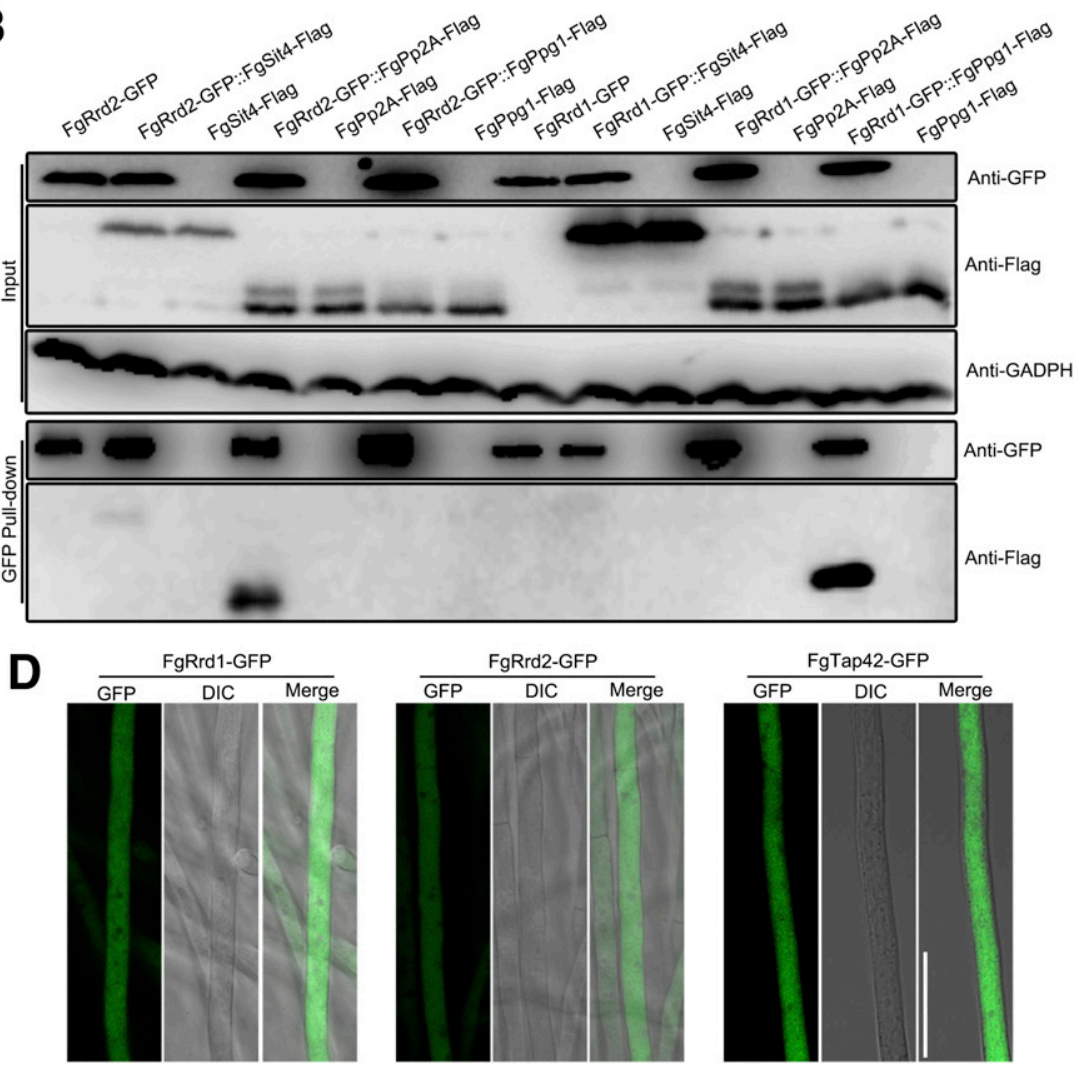

Fig. 2. FgRrd1 and FgRrd2 modulate the activity of FgPP2As (Fusarium graminearum type $2 \mathrm{~A}$ protein phosphatases) in F. graminearum. A, Comparisons of PP2A activity in wild-type PH-1, FgRRD1 deletion mutant $\triangle$ FgRrd1, FgRRD2 deletion mutant $\Delta$ FgRrd2, and complemented strains $\Delta$ FgRrd1-C and $\triangle$ FgRrd2C. The activity of PP2A was determined by measuring the dephosphorylation rate of the RII synthetic phosphopeptide substrate. Line bars in each column denote standard errors of three repeated experiments. Values on the bars followed by the same letter are not significantly different at $P=0.05$. B, Coimmunoprecipitation analysis of the interaction of FgRrd1 or FgRrd2 with three PP2As, namely, FgSit4, FgPp2A, or FgPpg1. Total proteins (input)

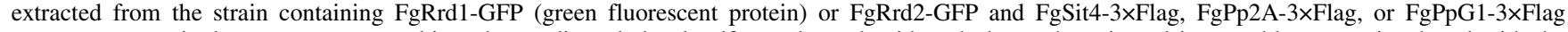
constructs or a single construct were subjected to sodium dodecyl sulfate-polyacrylamide gel electrophoresis and immunoblots were incubated with the polyclonal anti-Flag and monoclonal anti-GFP antibodies as indicated (upper panel). In addition, each protein sample was pulled down, using anti-GFP agarose, and was further detected with the monoclonal anti-GFP and polyclonal anti-Flag antibodies (lower panel). The protein samples were also detected with the monoclonal anti-GAPDH antibody as a reference. C, Yeast two-hybrid assays for identification of FgRrd1- and FgRrd2-interacting proteins. Serial dilutions of yeast cells (cells/milliliter), transferred with the bait and prey constructs indicated in the figure, were assayed for growth on yeast minimal synthetic defined base (SD) depleted of leucine, tryptophan, and histidine but supplemented with $3 \mathrm{mM} 3$-AT. A pair of plasmids, pGBKT7-53 and pGADT7, was used as a positive control. The pair of plasmids pGBKT7-Lam and pGADT7 was used as a negative control. The same set of yeast transformants was also assayed for $\beta$-galactosidase activity. D, The subcellular localization of FgRrd1-GFP, FgRrd2-GFP, and FgTap42-GFP. Bar $=20 \mu \mathrm{m}$. 
2D). Taken together, these results indicated that FgRrd1 and FgRrd2 interact with specific FgPP2A via the PP2A-associating protein FgTap42.

\section{FgRrd2 modulates methylation of FgPp2A.}

In humans, activation of the serine and threonine phosphatase PP2A by PTPA is associated with the PP2A methylesterase PME-1 (Longin et al. 2004; Van Hoof et al. 2005). To further explore the regulatory mechanism of FgPTPA, we detected the methylation level of $\mathrm{FgPp} 2 \mathrm{~A}$ in $F$. graminearum, using anti$\mathrm{Pp} 2 \mathrm{~A}$ and anti-Pp2A-Me antibodies. As shown in Figure 3A, the quantity of methylated $\mathrm{FgPp} 2 \mathrm{~A}$ was significantly decreased, while the total quantity of $\mathrm{FgPp} 2 \mathrm{~A}$ was increased in $\Delta \mathrm{FgRrd} 2$ but not in $\Delta \mathrm{FgRrd} 1$, the wild type, and complemented strains, indicating that only FgRrd2 is involved in regulating $\mathrm{FgPp} 2 \mathrm{~A}$ methylation (Fig. 3A).

To further understand the methylation of $\mathrm{FgPp} 2 \mathrm{~A}$, we were interested in identifying the methyltransferase that is responsible for methylation of FgPp2A. In-silico analysis identified a gene designated FgPPMI (FGSG_05090), encoding a putative protein phosphatase methyltransferase (PPM), by BLASTP search of the Fusarium genome database with $S$. cerevisiae PPM1 as a query. As expected, the deletion of $F g P P M 1$ led to dramatically decreased methylation and increased total quantity of FgPp2A (Fig. 3A). In addition, $\Delta \mathrm{FgPpm} 1$ and $\Delta \mathrm{FgRrd} 2$ displayed decreased sensitivity to caffeine compared with the wild type (Fig. 3B), indicating that

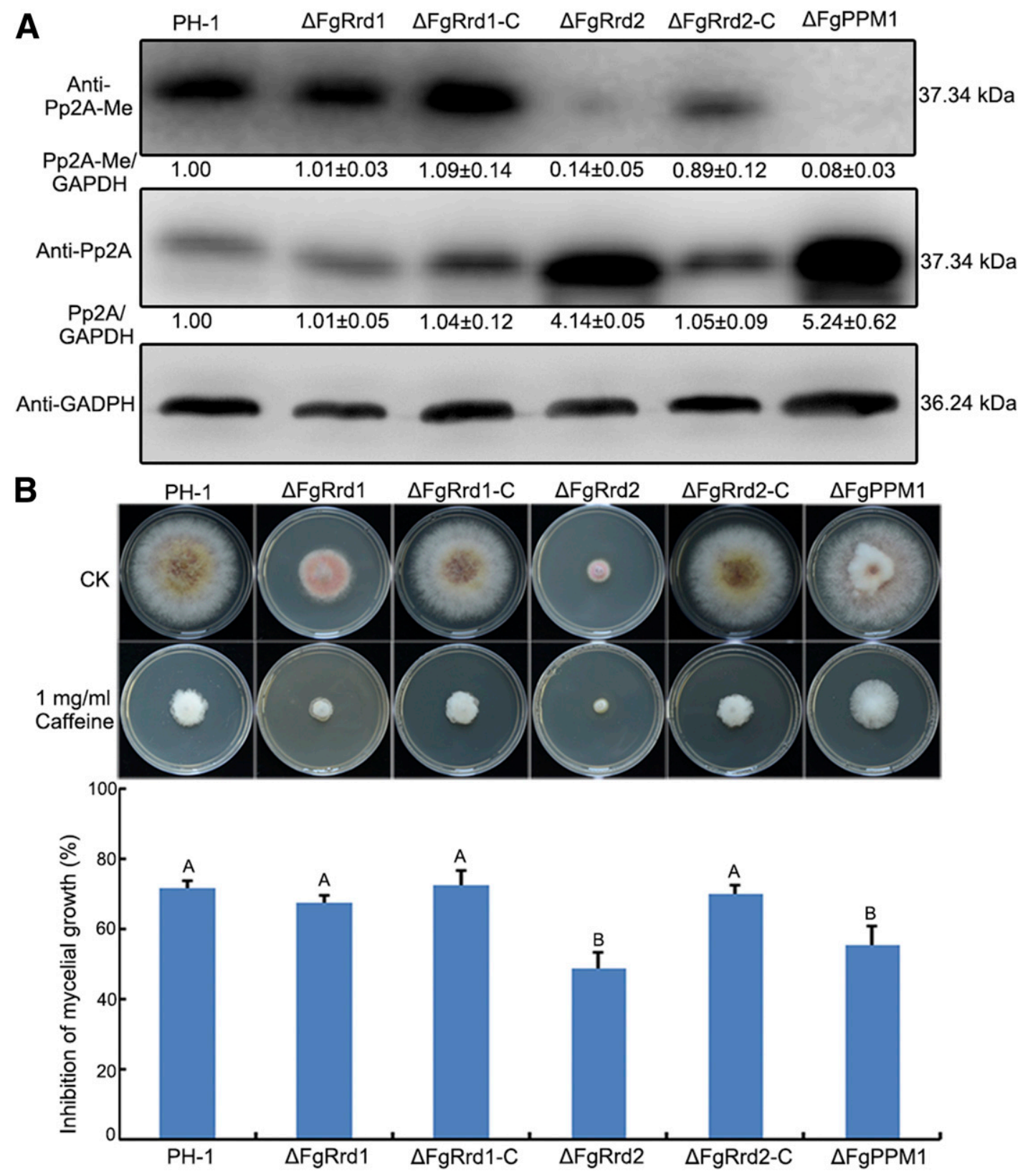

Fig. 3. FgRrd2 regulates the methylation of FgPp2A (Fusarium graminearum type $2 \mathrm{~A}$ protein phosphatase). A, Comparisons of FgPp2A methylation among $\mathrm{PH}-1, \Delta \mathrm{FgRrd} 1, \Delta \mathrm{FgRrd} 2, \Delta \mathrm{FgPpm} 1, \Delta \mathrm{FgRrd} 1-\mathrm{C}$, and $\Delta$ FgRrd2-C. Pp2A and methylated Pp2A were detected using the anti-Pp2A and antimethylation Leu309 of FgPp2A (anti-Pp2A-Me) antibodies, respectively. The intensities of the Western blotting bands were quantified with the program IMAGE QUANT TL. The intensity of the Pp2A methylation or Pp2A band for each strain is relative to that of GAPDH band. $\mathbf{B}, \Delta \mathrm{FgRrd} 2$ and $\Delta \mathrm{FgPpm} 1 \mathrm{showed}$ increased resistance to caffeine. A 5-mm mycelial plug of PH-1, $\Delta \mathrm{FgRrd} 1, \Delta \mathrm{FgRrd} 2, \Delta \mathrm{FgPpm} 1$, and the complemented strains $\Delta \mathrm{FgRrd} 1-\mathrm{C}$ and $\Delta \mathrm{FgRrd} 2-\mathrm{C}$ was inoculated on potato dextrose agar amended without or with $1 \mu \mathrm{g}$ of caffeine per milliliter. Photos were taken after incubation at $25^{\circ} \mathrm{C}$ for 3 days (upper panel), and colony diameter was calculated for each strain (lower panel). Bars denote standard errors from three independent experiments. Values on the bars followed by the same letter are not significantly different at $P=0.05$. 
the methylated FgPp2A is associated with caffeine sensitivity. Taken together, these results indicate that PPM1 as well as FgRrd2 is responsible for methylation of FgPp2A in F. graminearum.

\section{Involvement of FgRrd1 and FgRrd2}

in mycelial growth, asexual, and sexual development.

The $\Delta$ FgRrd 1 and $\Delta F g R r d 2$ mutants grew dramatically slower than the wild-type progenitor on potato dextrose agar (PDA), minimal medium (MM), and wheat-head medium (Fig. 4A and B). The growth defects of $\Delta \mathrm{FgRrd} 1$ and $\Delta \mathrm{FgRrd} 2$ were restored by genetic complementation with the wild-type $F g R R D 1$ and FgRRD2, respectively (Fig. 4A and B). Conidiation assays showed that $\Delta \mathrm{FgRrd} 1$ and $\Delta \mathrm{FgRrd} 2$ produced significantly less conidia than the wild type after 4 days of incubation in mung bean liquid (MBL) medium (Fig. 4C and D). Moreover, microscopic examination revealed that $\Delta \mathrm{FgRrd} 1$ and $\Delta \mathrm{FgRrd} 2$ produced
A

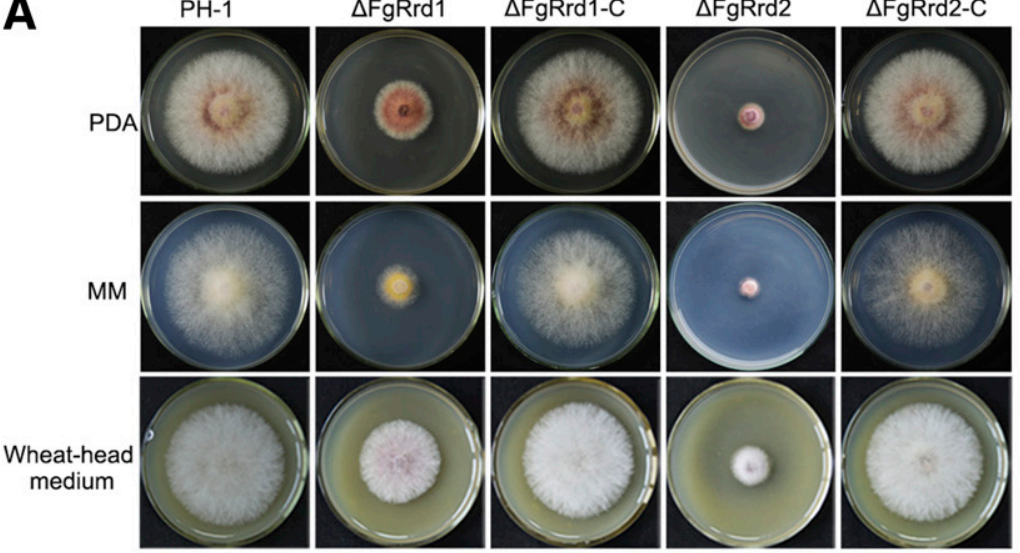

B
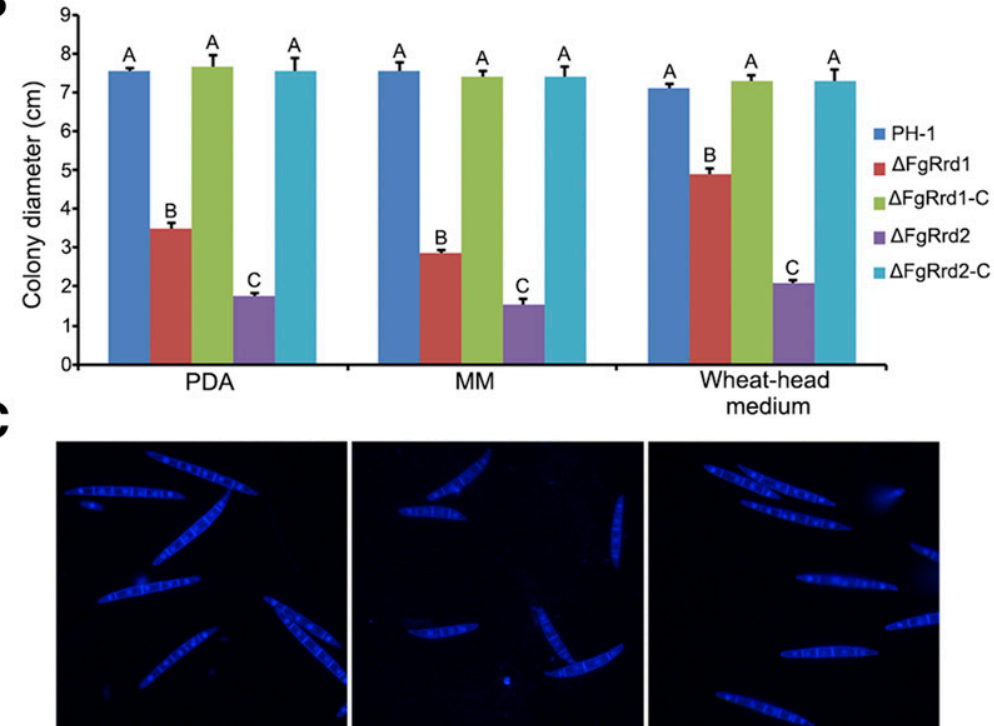

$\mathrm{PH}-1$

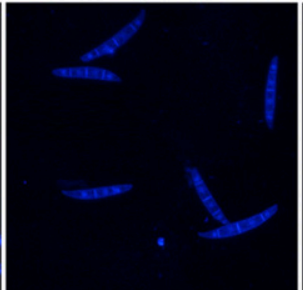

$\Delta$ FgRrd1

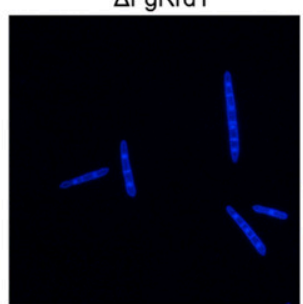

$\triangle \mathrm{FgRrd} 2$

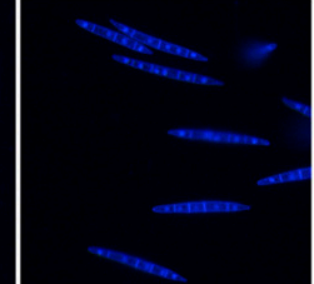

$\triangle \mathrm{FgRrd1}-\mathrm{C}$

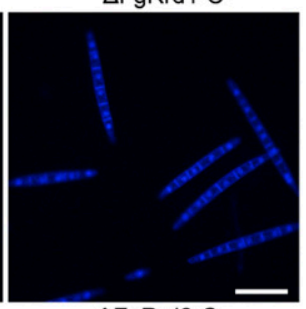

$\mathbf{E}$

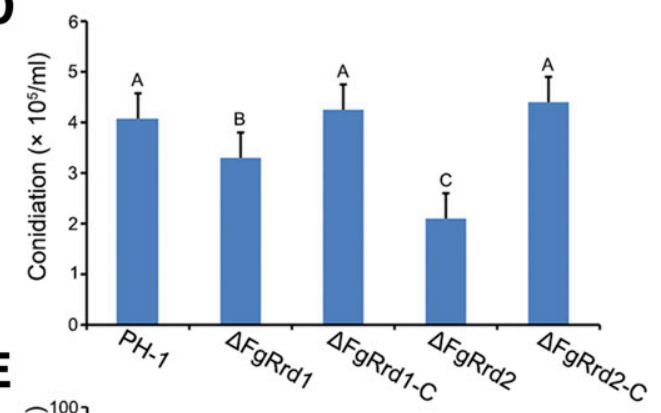

$\mathbf{F}$
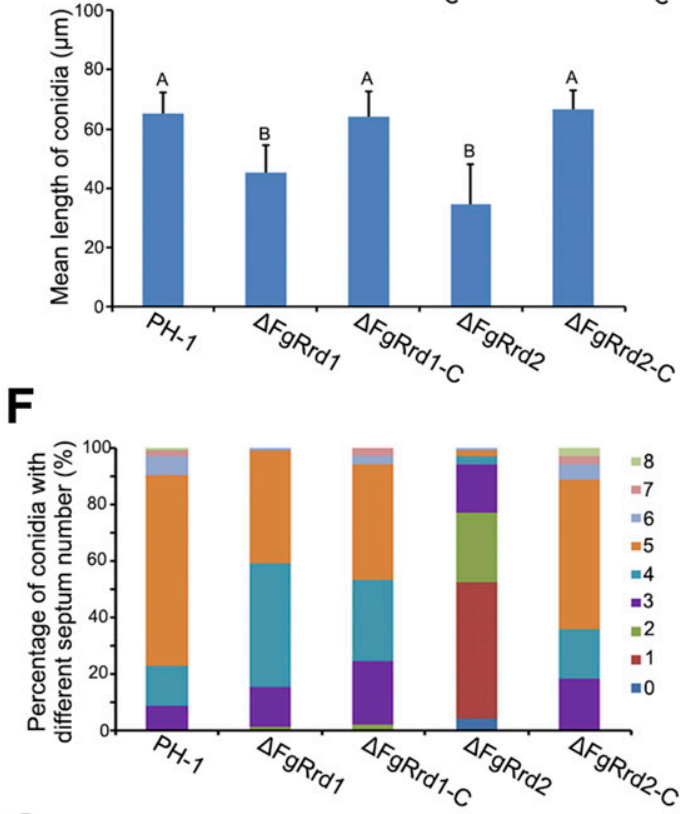

$\mathbf{G}$

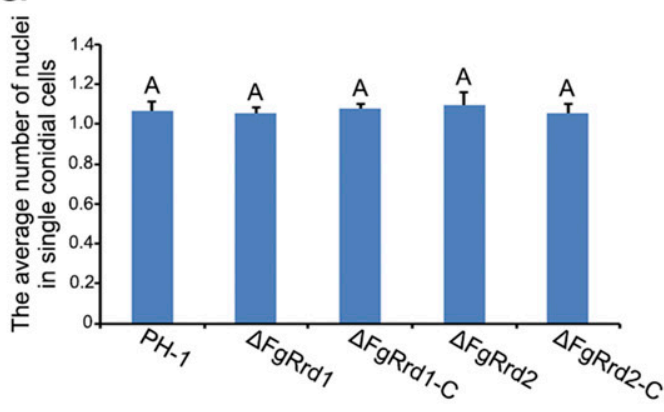

Fig. 4. Involvement of FgRrd1 and FgRrd2 in regulating vegetative growth and conidiation in Fusarium graminearum. A, Colony morphology of the wild-type $\mathrm{PH}-1, \Delta \mathrm{FgRrd} 1$, and $\Delta$ FgRrd2, and the complemented transformants $\Delta$ FgRrd1-C and $\Delta$ FgRrd2-C on potato dextrose agar (PDA), minimal medium (MM) and wheat-head media at $25^{\circ} \mathrm{C}$ for 3 days. B, Colony diameter of each strain cultured on PDA, MM, or wheat-head medium at $25^{\circ} \mathrm{C}$ for 3 days. Line bars in each column denote standard errors of three repeated experiments. Values on the bars followed by the same letter for each medium are not significantly different at $P=0.05$. C, Differential interference contrast images of conidia stained with calcofluor white and 4',6-diamidino-2-phenylindole were captured with electron microscopy. Bar $=20 \mu \mathrm{m}$. D, Conidia were quantified using a hemacytometer after incubation in mung bean liquid for 4 days. Line bars in each column denote standard errors of three repeated experiments. Bars with the same letter indicate no significant difference at $P=0.05$. E, Comparisons in mean conidial length among the above strains. A total of 200 conidia were examined for each strain in each experiment. Line bars in each column denote standard errors of three repeated experiments. Values on the bars followed by the same letter for each medium are not significantly different at $P=0.05$. F, Comparisons in septum number of conidia among the above strains. A total of 200 conidia were examined for each strain in each experiment. $\mathbf{G}$, Comparisons in nucleus number of conidia among the above strains. A total of 200 conidia were examined for each strain in each experiment. Line bars in each column denote standard errors of three repeated experiments. Values on the bars followed by the same letter are not significantly different at $P=0.05$. 
shorter conidia with fewer septa in comparison with those of the wild type (Fig. 4C, E, and F). The mean lengths of $\Delta \mathrm{FgRrd} 1$ and $\Delta$ FgRrd2 were reduced by 31 and $47 \%$, respectively, compared with that of the wild-type conidia (Fig. 4E). Most conidia of PH-1 had five or six septa, however, $59 \%$ conidia of $\Delta$ FgRrd1 and $97 \%$ conidia of $\Delta$ FgRrd2 have fewer than five septa (Fig. 4F). In addition, deletion of $F g R R D 1$ and $F g R R D 2$ did not cause detectable changes in nucleus number in each conidial cell (Fig. 4C and G) and in conidial germination (Supplementary Fig. S2).

As a homothallic fungus, sexual production plays important roles in the infection cycle of $F$. graminearum (Min et al. 2012; Trail 2009). Previous study has shown that FgPpg1 and FgSit4 are required to produce perithecia on carrot agar ( $\mathrm{Yu}$ et al. 2014). To characterize the function of FgRrd1 and FgRrd2 in sexual development, we determined the ability of the mutants to form perithecia on carrot agar. As shown in Figure 5A and B, both $\Delta \mathrm{FgRrd} 1$ and $\Delta \mathrm{FgRrd} 2$ were sexually sterile, whereas the wild type and the complemented strains produced mature perithecia containing normal asci. This observation was further confirmed by determining the expression levels of six sexual development-related genes (FGSG_00362, FgKIC1; FGSG_ 00573; FGSG_01058,FgPUK1; FGSG_04480; FGSG_04947, FgCAK1; FGSG_08701, FgHSL1), since expression of these genes was highly up-regulated during sexual development and deletion mutants of these genes displayed serious defects in sexual reproduction ability (Qi et al. 2006; Son et al. 2011; Wang et al. 2011). Quantitative reverse transcription (qRT)-PCR assays showed that these six sexual reproduction-related genes were down-regulated significantly in these two mutants (Fig. 5C). Taken together, these results demonstrate that FgRrd1 and FgRrd2 regulate vegetative growth, asexual, and sexual development in F. graminearum.

FgRrd1 and FgRrd2 are indispensable for lipid droplet biogenesis in F. graminearum.

Previous study with $F$. graminearum has shown that deletion of FgSIT4 or FgPPG1 prevented the accumulation of lipid droplets under the treatment with rapamycin (Yu et al. 2014). Thus, we were interested in checking the effect of FgPTPA deletion on lipid droplet biosynthesis. Nile Red and Bodipy staining assays showed that only a few lipid droplets were observed in the hyphae of $\Delta \mathrm{FgRrd} 1$ and $\Delta \mathrm{FgRrd} 2$ mutants treated with $0.25 \mu \mathrm{g}$ of rapamycin per milliliter, whereas many lipid droplets were detected in the wild-type hyphae with the same treatment (Fig. 6). These results indicate that the formation of lipid droplets in $F$. graminearum triggered by rapamycin is dependent on FgRrd1 and FgRrd2.

\section{FgRrd1 and FgRrd2 regulate transcription of DON biosynthesis genes via interacting with RNA polymerase II.}

The mycotoxin DON is an important virulence factor of F. graminearum (Desjardins et al. 1996; Proctor et al. 1995a; Seong et al. 2008). We therefore determined DON production by each mutant. After incubation on wheat kernels for 20 days, $\Delta$ FgRrd 1 and $\Delta$ FgRrd2 produced undetectable DON (Fig. 7A).

A

$\mathrm{PH}-1$

$\Delta$ FgRrd1

$\Delta$ FgRrd1-C

$\Delta \mathrm{FgRrd} 2$

$\Delta$ FgRrd2-C
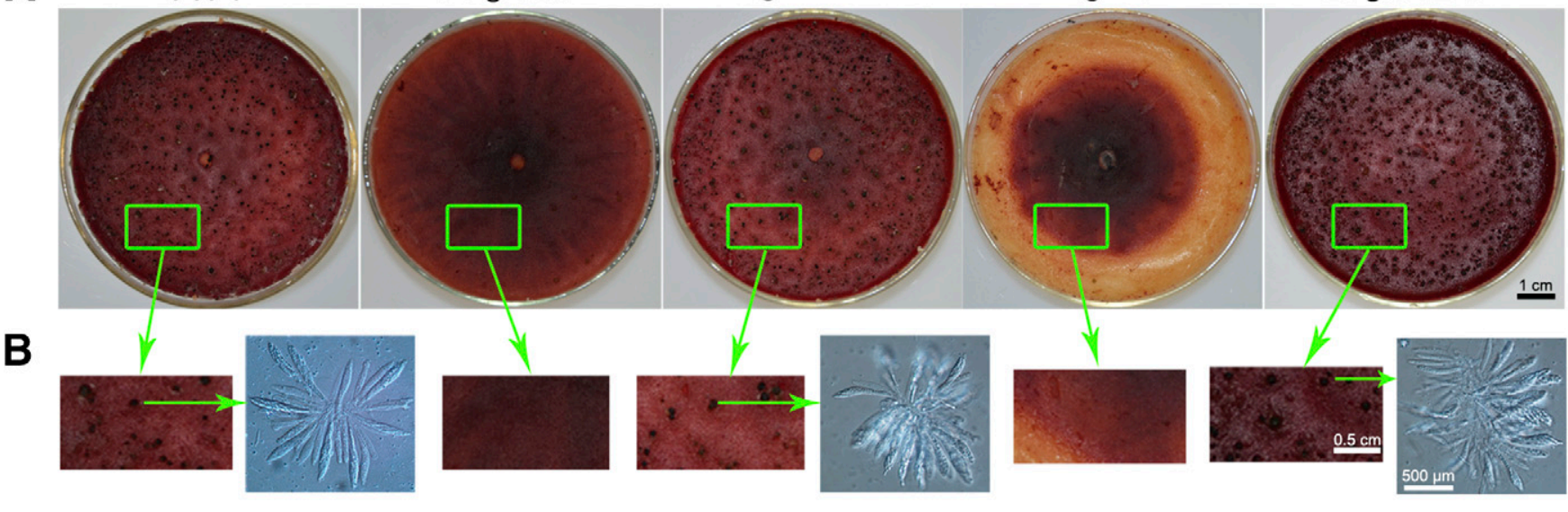

C

FgKIC1

FGSG_00573

FgPUK1

FGSG_04480

FgCAK1

FgHSL1
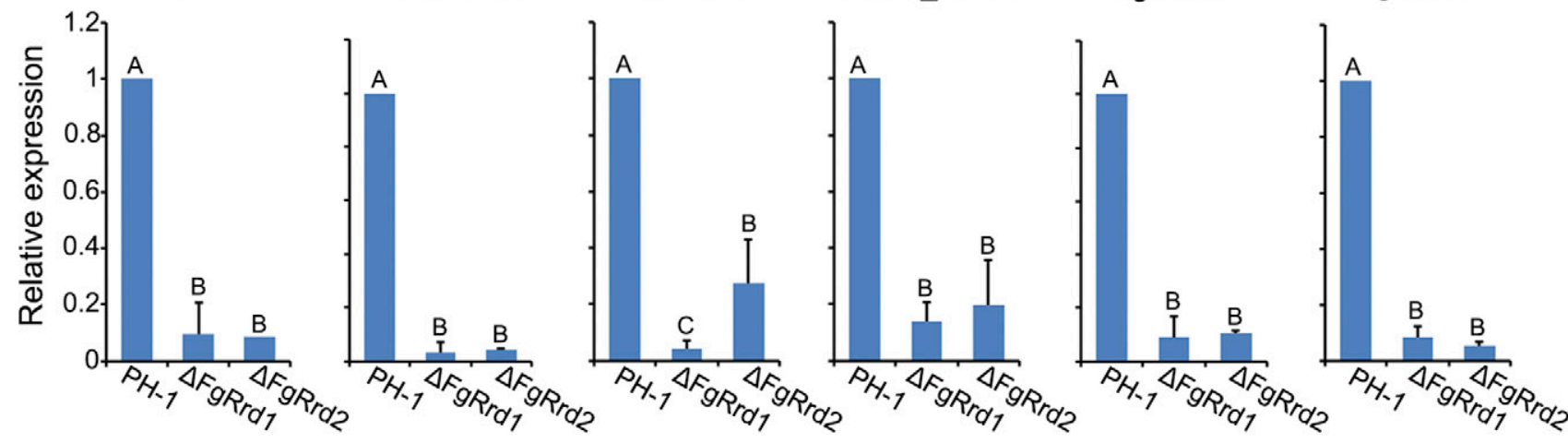

Fig. 5. Effects of FgRRD1 or FgRRD2 deletion on sexual development in Fusarium graminearum. A, Each mutant and its corresponding complementation strain were grown on carrot agar for induction of perithecial formation. Bar $=500 \mu \mathrm{m}$. B, A closeup view of perithecia and the examination of ascus and ascospore development. C, Comparisons of the transcript levels of sexual development-related genes in the wild-type PH-1 and the mutants $\Delta$ FgRrd1 and $\Delta$ FgRrd2, after culture on carrot medium for 7 days. The relative expression level of each gene was analyzed with the $2^{-\Delta \Delta C t}$ method, with the $A C T I N$ gene as the internal control for normalization. The expression level of each gene in the wild type was referred to as 1 . Line bars in each column denote standard errors of three repeated experiments. Values on the bars followed by the same letter in each graph are not significantly different at $P=0.05$. 
qRT-PCR assays showed that the expression levels of two DON biosynthesis genes, FgTRI5 and FgTRI6, in these two mutants were dramatically decreased in comparison with those in the wild type (Fig. 7B). Interestingly, we found that, in comparison with $\Delta$ FgSit4, the defects in DON production of $\Delta$ FgRrd1 and $\triangle \mathrm{FgRrd} 2$ were more serious (Fig. 7A). Moreover, the expression levels of FgTRI5 and FgTRI6 in $\triangle$ FgRrd1 and $\triangle$ FgRrd2 were significantly lower than those in $\Delta$ FgSit4 and $\Delta$ FgPpg 1 (Fig. 7B). These results suggest that FgRrd1 and FgRrd2 may have the additional function of regulating DON biosynthesis in F. graminearum.

In the budding yeasts, Rrd1 functions as a peptidyl prolyl cis/trans isomerase (PPIase), and has been found to isomerize RNA polymerase II, subsequently regulates gene expression in response to diverse conditions (Jouvet et al. 2010; Poschmann et al. 2011; Sen et al. 2014). Therefore, we conducted Co-IP assays to detect the interaction of FgRrd1 and FgRrd2 with RNA polymerase II, using anti-GFP and the antiphosphorylated carboxy-terminal domain (CTD) of RNA polymerase II (antiphospho-CTD) antibodies. As shown in Figure 7C, both FgRrd1 and FgRrd2 physically interacted with RNA polymerase II in the Co-IP assays. Using chromatin immunoprecipitation (ChIP) and qRT-PCR analysis, we further found that the enrichment of RNA polymerase II at the promoters of $12 \mathrm{FgTRI}$ genes in $\triangle \mathrm{FgRrd} 1$ and $\Delta$ FgRrd2 were significantly lower than those in the wild type (Fig. 7D). In addition, we found that FgPp2A, FgPpg1, and FgSit4 did not interact with RNA polymerase II by Co-IP analyses (Supplementary Fig. S3). These results indicate that FgRrd1 and $\mathrm{FgRrd} 2$ could regulate transcription of genes via interacting with RNA polymerase II, which is independently on PP2A in $F$. graminearum.

Since DON as an important virulence factor plays key roles in the extension of $F$. graminearum in the host plant (Proctor et al. 1995b), virulence of $\Delta \mathrm{FgRrd} 1$ and $\Delta \mathrm{FgRrd} 2$ was evaluated. After 3 weeks of point-inoculation of conidial suspension on flowering wheat head, $\Delta \mathrm{FgRrd} 1$ and $\Delta \mathrm{FgRrd} 2$ caused blight symptoms only in the inoculated spikelets, whereas the wildtype PH-1 and complemented strains caused typical blight symptoms on wheat heads under the same conditions (Fig. 7E). This result agrees with the finding that FgRrd1 and FgRrd2 are indispensable for DON biosynthesis in F. graminearum.

\section{FgRrd2 modulates the response of $F$. graminearum} to phenylpyrrole fungicide fludioxonil.

To determine whether FgRrd1 and FgRrd2 are involved in coping with various environmental stresses, serial dilutions of conidial suspension of each mutant were spotted on PDA amended with fungicides fludioxonil, carbendazim, tebuconazole, or phenamacril or on MM supplemented with cell-wall or oxidative stress. We found that $\Delta \mathrm{FgRrd} 2$ became more sensitive to phenylpyrrole fungicide fludioxonil but not to other stresses and the growth inhibition of $\Delta \mathrm{FgRrd} 1$ under each stress is undistinguishable from that of the wild type (Fig. 8A; Supplementary Fig. S4). Since the phenylpyrrole fungicides have been found to activate the high-osmolarity glycerol (HOG) pathway in several fungal pathogens (Kojima et al. 2002), we were also interested in analyzing the phosphorylation profiling of FgHog 1 in $\Delta \mathrm{FgRrd} 2$, which is the key MAPK in the F. graminearum HOG pathway (Hou et al. 2002). As shown in Figure $8 \mathrm{~B}$ and $\mathrm{C}$, the phosphorylation level of FgHog1 was significantly upregulated in $\Delta \mathrm{FgRrd} 2$, indicating that $\mathrm{FgRrd} 2$ might negatively regulate the phosphorylation of FgHog1 in F. graminearum.

\section{DISCUSSION}

PTPAs as a class of PP2A activators play important roles in regulating the activity of PP2A catalytic subunits (Fellner et al. 2003; Leulliot et al. 2006). Previous studies with the budding yeasts have found the different PTPAs are responsible for activating specific PP2As (Van Hoof et al. 2005; Zheng and Jiang 2005). There are five type $2 \mathrm{~A}$ protein phosphatases Pph21, Pph22, Pph3, Ppg1, and Sit4 in S. cerevisiae. Rrd1 preferentially binds to Pph3, Ppg1, and Sit4, whereas Rrd2 is inclined to interact with Pph21 and Pph22 (Van Hoof et al. 2005; Zheng and Jiang 2005). However, the corresponding relationships of PTPAs and PP2As in F. graminearum differ from those in the budding yeasts. In this study, Co-IP assays showed that FgRrd1 only interacts with $\mathrm{FgPpg} 1$, whereas FgRrd2 binds mainly to FgPp2A (the ortholog of Pph21 or Pph22) (Fig. 2B). More importantly, we found, for the first time, that FgRrd1 and FgRrd2 both interact with PP2A-associating protein FgTap42 directly, via $\mathrm{Y} 2 \mathrm{H}$ assays, which indicate that FgPTPA, FgTap42, and FgPP2A phosphatase form ternary complexes and FgTap42 serves as a bridge between FgPTPA and FgPP2A.

The detailed molecular mechanism of PP2A activity regulated by PTPA is not clear in fungi. Previous studies showed PTPA in mammalian and yeasts may modulate the activity of PP2A via changing PP2A conformation, since PTPA belongs to a peptidyl prolyl cis/trans isomerase (Jordens et al. 2006). However, we surprisingly found that lack of $F g R R D 2$ led to a significantly decreased methylation level of $\mathrm{FgPp} 2 \mathrm{~A}$ that is associated with response to caffeine (Fig. 3A and B; Supplementary Fig. S5). In humans, PTPA was found to interplay with

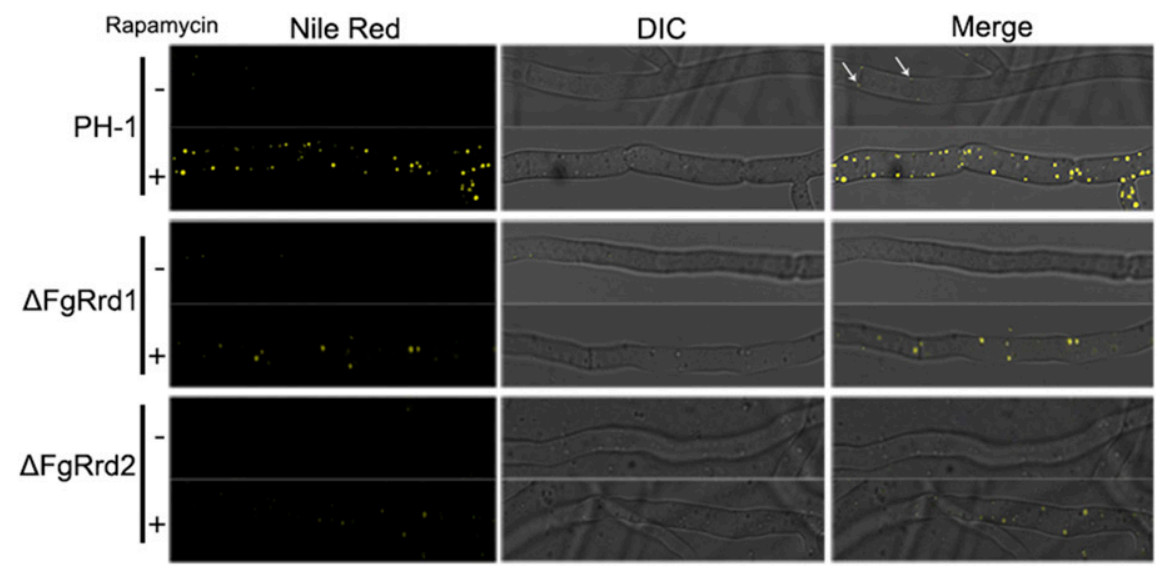

Fig. 6. Deletion of FgRRD1 or FgRRD2 impedes lipid droplet accumulation induced by rapamycin treatment. After treatment with $0.25 \mu$ g of rapamycin per milliliter for $5 \mathrm{~h}$, lipid droplet accumulation in the mycelia of deletion mutants $\Delta$ FgRrd 1 and $\Delta$ FgRrd 2 were stained with Nile Red (lipid droplet was indicated with white arrows). The wild-type $\mathrm{PH}-1$ was used as the positive control. Bar $=20 \mu \mathrm{m}$. 
A

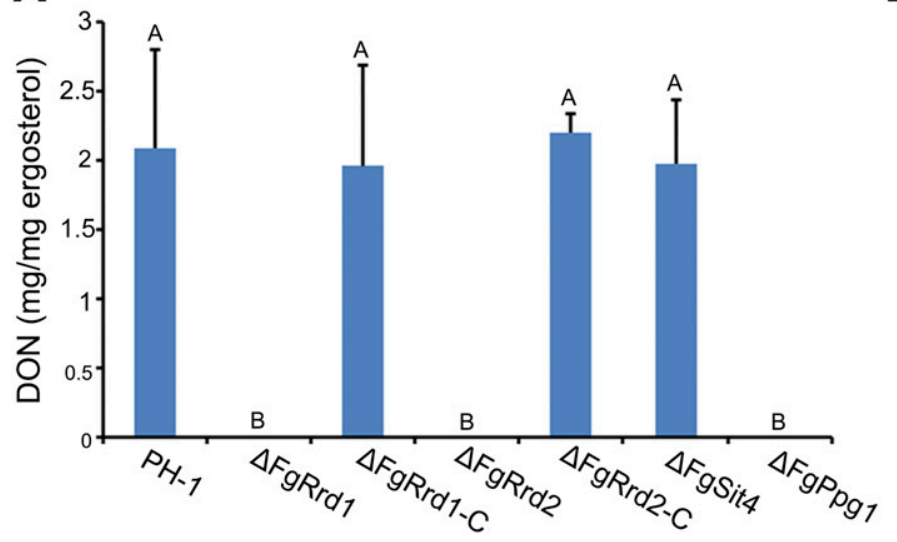

B

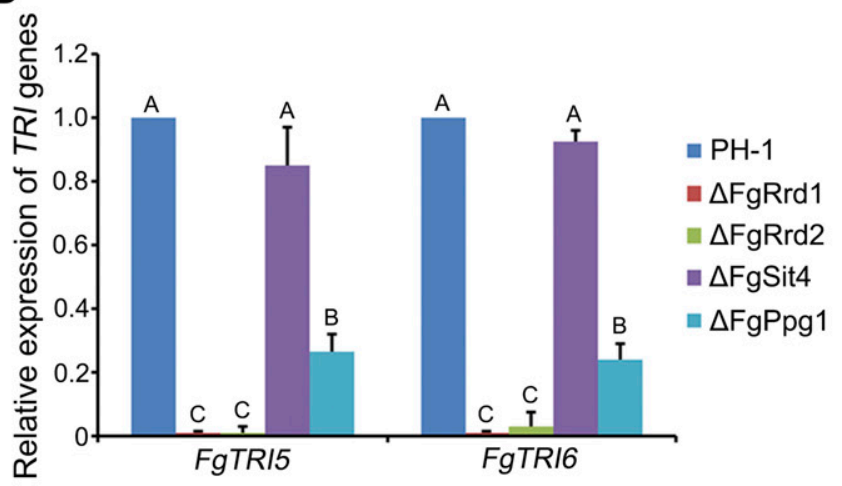

C

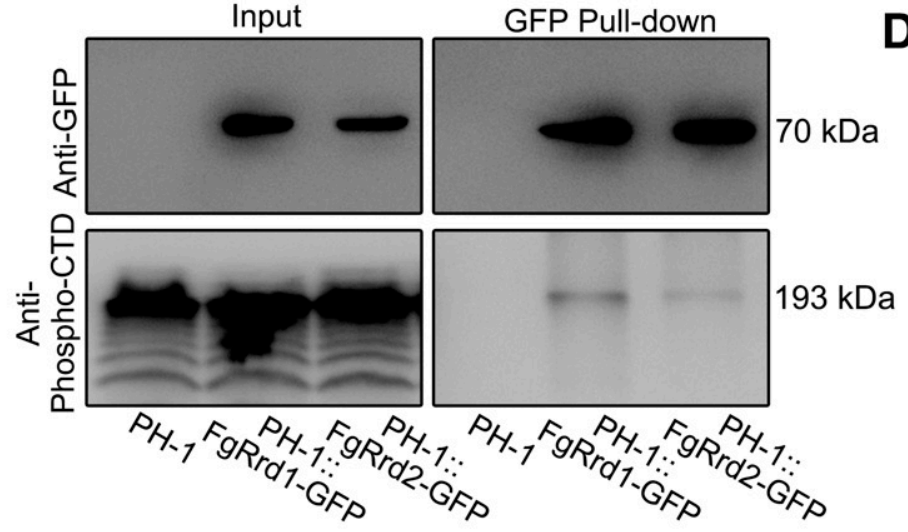

D

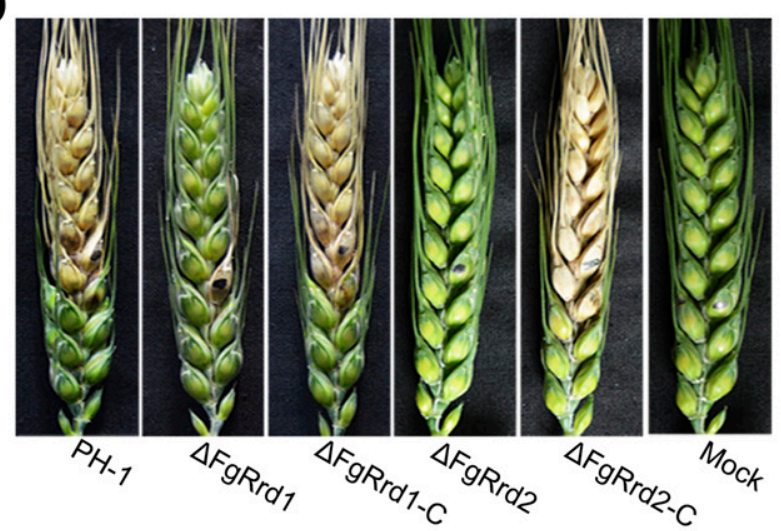

E

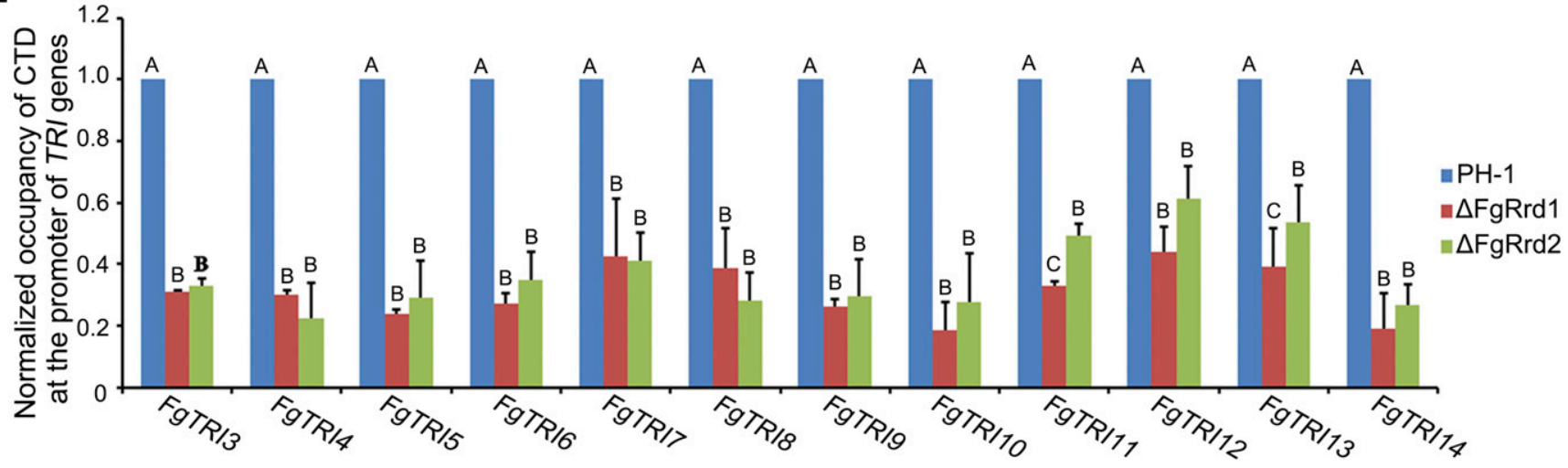

Fig. 7. FgRrd1 and FgRrd2 regulate the enrichment of RNA polymerase II at the promoters of FgTRI genes in Fusarium graminearum. A, The amounts of deoxynivalenol (DON) (per milligram of fungal ergosterol) produced by the wild-type $\mathrm{PH}-1, \Delta \mathrm{FgRrd} 1, \Delta \mathrm{FgRrd} 2, \Delta \mathrm{FgSit} 4$ and $\Delta \mathrm{FgPpg} 1$, and the complemented strains $\Delta$ FgRrd1-C and $\Delta$ FgRrd2-C in infected wheat kernels. Line bars in each column denote standard errors of three replicated experiments. Values on the bars followed by the same letter are not significantly different at $P=0.05$. B, Comparisons of the transcript levels of FgTRI5 and FgTRI6 in PH-1, and $\Delta \mathrm{FgRrd} 1, \Delta \mathrm{FgRrd} 2, \Delta \mathrm{FgSit} 4$, and $\Delta \mathrm{FgPpg} 1$ cultured in the trichothecene biosynthesis induction (TBI) medium for 2 days at $25^{\circ} \mathrm{C}$ in the dark. The relative expression level of each gene was analyzed with the $2^{-} \Delta \Delta^{\mathrm{Ct}}$ method with the ACTIN gene as the internal control for normalization. The expression level of each gene in the wild type was referred to as 1 . Line bars in each column denote standard errors of three repeated experiments. Values on the bars followed by the same letter are not significantly different at $P=0.05$. C, RNA polymerase II physically interacts with FgRrd1 and FgRrd2 in a coimmunoprecipitation assay. Total proteins (input) extracted from the strain bearing a single FgRrd1-GFP (green fluorescent protein) or FgRrd2-GFP construct and cultured in the TBI medium for 2 days at $25^{\circ} \mathrm{C}$ in the dark were subjected to sodium dodecyl sulfate-polyacrylamide gel electrophoresis and immunoblots were incubated with the monoclonal antibodies anti-GFP and antiphosphorylated carboxy-terminal domain of RNA polymerase II (anti-phospho-CTD) (upper panel). In addition, each protein sample was pulled down using the anti-GFP agarose and was further detected with the monoclonal anti-GFP monoclonal anti-phospho-CTD antibody (lower panel). D, Flowering wheat heads were point-inoculated with a conidial suspension at $10^{5}$ conidia/ml of each strain, and infected wheat heads were photographed three weeks after inoculation. E, The recruitment of RNA polymerase II at the $5^{\prime}$ region of FgTRI genes in $\Delta$ FgRrd1 and $\Delta$ FgRrd2 were dramatically reduced in comparisons with those in PH-1 after incubation in TBI medium for 2 days. Relative enrichment values were calculated by dividing the ratio of band intensity for immunoprecipitated DNA of each gene with the ratio of intensity for total input DNA. Line bars in each column denote standard errors of three repeated experiments. Values on the bars followed by the same letter for each gene are not significantly different at $P=0.05$. 
PP2A carboxyl methylation-related eznzyme, LCMT1 (leucine carboxyl methyltransferase 1) and PME-1 (PP2A methylesterase) (Hombauer et al. 2007; Sents et al. 2013). However, the direct effect of PTPA on methylation of PP2A has not yet been documented. To our knowledge, it is the first report that a PTPA is involved in regulating PP2A methylation.

In $F$. graminearum, FgRrd 1 and $F g R r d 2$ regulate lipid droplet accumulation induced by rapamycin, and the counterparts of $S$. cerevisiae modulate the sensitivity to rapamycin (Rempola et al. 2000), indicating PTPA orthologs are involved in the TOR pathway in both yeast and filamentous fungi. However, we also found species-specific functions of PTPA in the budding yeasts and F. graminearum. First, although the FgRrd1 and FgRrd2 mutants both displayed defects in sexual reproduction and virulence, the FgRrd2 mutant of $F$. graminearum displayed more serious defects in mycelial growth and conidial development than the FgRrd1 mutant, indicating that FgRrd2 plays more important roles than FgRrd1. Consistently, the FgRrd2interacting protein $\mathrm{Pp} 2 \mathrm{~A}$ but not FgRrd1-interacting protein FgPpg1 is essential for $F$. graminearum growth (Yu et al. 2014). In contrast, the $S$. cerevisiae RRDI deletion mutant shows several pleiotropic defects in growth rate and cell viability under various conditions, whereas the RRD2 mutant is undistinguishable from the wild type in growth rate (Rempola et al. 2000). Second, Rrd1 and Rrd2 could control cell division via modulating the cell cycle during G1 or M phase in S. cerevisiae (Van Hoof et al. 2000, 2001). However, FgRrd1 and FgRrd2 were not involved in the regulation of cell division as $\Delta \mathrm{FgRrd} 1$ and $\Delta \mathrm{FgRrd} 2$ conidial cells possess normal nucleus number (Fig. 4C). These results indicate that PTPAs possess speciesspecific functions in different fungi.

More recently, several studies reported that Rrd1 but not Rrd2 interacts with RNA polymerase II and further alters the conformation of the CTD of RNA polymerase II, which leads to transcriptional change of genes regulated by rapamycin or the TOR pathway (Jouvet et al. 2010; Poschmann et al. 2011; Sen et al. 2014). However, it remains unclear whether the function of Rrd1 in conformation of RNA is dependent on PP2A. In the current study, we found both FgRrd1 and FgRrd2 interact with CTD of RNA polymerase II. Further, we found that the enrichment of CTD at the $5^{\prime}$ of DON biosynthesis genes was dramatically reduced in $\triangle \mathrm{FgRrd} 1$ and $\Delta \mathrm{FgRrd} 2$. Moreover, deletion of FgRRD1 or FgRRD2 caused seriously downregulated expression levels of FgTRI genes and no detectable DON production (Fig. 7A and B).

A

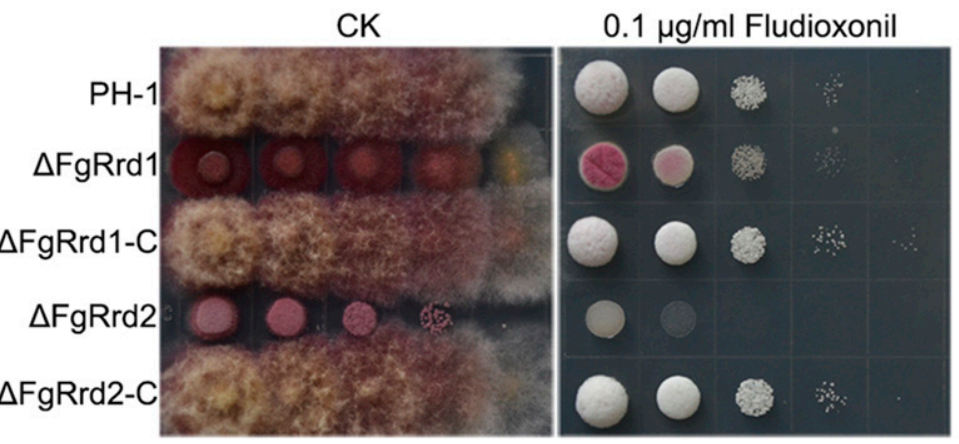

B

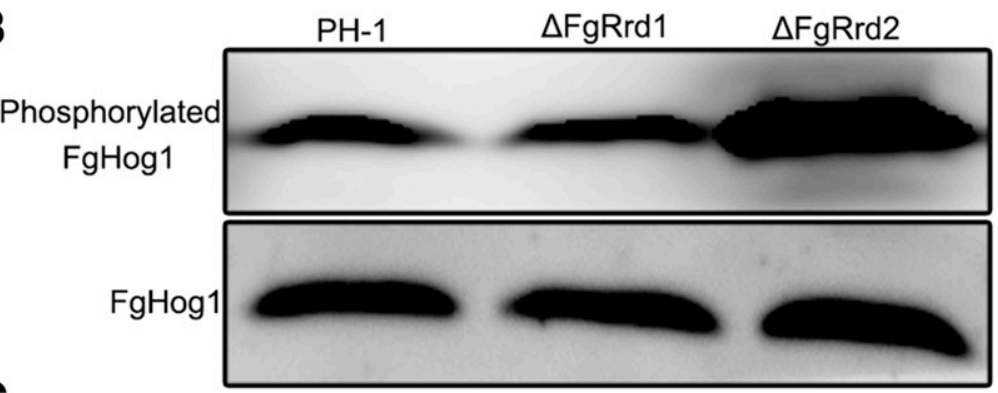

C

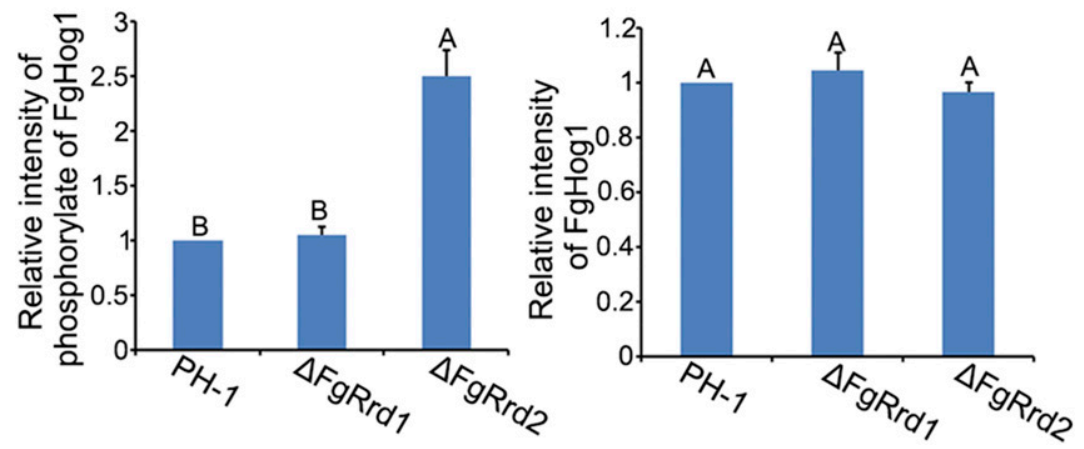

Fig. 8. FgRrd2 is involved in the response of Fusarium graminearum to the phenylpyrrole fungicide fludioxonil. A, Sensitivity of the wild-type PH-1, $\Delta$ FgRrd1, and $\Delta \mathrm{FgRrd} 2$ and the complemented strains $\Delta \mathrm{FgRrd} 1-\mathrm{C}$ and $\Delta \mathrm{FgRrd} 2-\mathrm{C}$ to fludioxonil. Serial dilutions of conidial suspension of each strain were spotted on potato dextrose agar (PDA) and PDA supplemented with $0.1 \mu \mathrm{g}$ of fludioxonil per milliliter. Images were taken after 2 days of incubation at $25^{\circ} \mathrm{C}$. B, Comparisons of phosphorylation of FgHog1 among PH-1, $\Delta \mathrm{FgRrd} 1$, and $\Delta \mathrm{FgRrd} 2$. Phosphorylated FgHog1 and FgHog1 were detected by the antibody against dually phosphorylated p38 (Thr180/Tyr182) and an anti-Hog1 antibody, respectively. C, The intensities of the Western blotting bands were quantified with the program IMAGE QUANT TL. The intensity of the FgHog1 band in each mutant is relative to the amount of FgHog1 in the wild-type PH-1. Bars denote standard errors from three independent experiments. Values on the bars followed by the same letter are not significantly different at $P=0.05$. 
More importantly, three FgPP2As were not found to interact with RNA polymerase II. Taken together, these results indicate that both FgRrd1 and FgRrd2 have the ability to regulate the transcription of secondary metabolic genes in $F$. graminearum, which is independent of FgPP2As.

In the budding yeasts, the synthetic lethality of deletion of both Rrd1 and Rrd 2 could be suppressed by the viable allele (SSD1-v1) of the SSD1 (a suppressor of Sit4) (Fellner et al. 2003; Kaeberlein et al. 2004; Rempola et al. 2000; Van Hoof et al. 2000 , 2001). Given that Sit4 is involved in CWI, S. cerevisiae Rrd1 and Rrd2 were thought to be associated with the CWI pathway. On the other hand, previous studies showed that $F$. graminearum PP2A phosphatases FgSit4 and FgPpg1 positively regulate phosphorylation of FgMgv1 via interaction with FgMsg5, a negative regulator of the CWI pathway (Flández et al. 2004; Yu et al. 2014). However, our study found that both FgRrd1 and FgRrd2 deletion mutants did not show changed sensitivity to the cell wall-damaging agents Congo red, Calcofluor white (CFW), and sodium dodecyl sulfate (SDS) in comparison with the wild type. Consistently, Western blotting assay showed that deletion of FgRRD1 or FgRRD2 did not alter the phosphorylation level of MAPK FgMgv1 in the CFW. The results indicated that the reduced activity of FgPP2As in $\Delta$ FgRrd1 and $\Delta \mathrm{FgRrd} 2$ might still be sufficient to maintain CWI in F. graminearum. In addition, S. cerevisiae Rrd1 and Rrd2 were involved in response to DNA damage, caffeine, or transcriptional stress (Douville et al. 2004; Poschmann et al. 2011; Rempola et al. 2000). However, determining responses to multiple stresses, only FgRrd2 exhibited increased sensitivity to phenylpyrrole fungicide and both FgRrd1 and FgRrd2 showed an undetectable change to other fungicides and oxidative stress compared with the wild type.

\section{MATERIALS AND METHODS}

\section{Strains and culture conditions.}

F. graminearum wild-type strain $\mathrm{PH}-1$ was used as a parental strain for transformation experiments. The wild-type strain and transformants generated in this study were grown on PDA ( $200 \mathrm{~g}$ of potato, $20 \mathrm{~g}$ of dextrose, $20 \mathrm{~g}$ of agar, and 1 liter of water), $\mathrm{MM}\left(10 \mathrm{mM} \mathrm{K} \mathrm{HPO}_{4}, 10 \mathrm{mM} \mathrm{KH_{2 }} \mathrm{PO}_{4}, 4 \mathrm{mM}\right.$ (NH4) ${ }_{2} \mathrm{SO}_{4}, 2.5 \mathrm{mM} \mathrm{NaCl}, 2 \mathrm{mM} \mathrm{MgSO}, 0.45 \mathrm{mM} \mathrm{CaCl}_{2}$, $9 \mathrm{mM} \mathrm{FeSO}_{4}$, and $10 \mathrm{mM}$ glucose, $\mathrm{pH}$ 6.9), or wheat-head medium (200 $\mathrm{g}$ of ground fresh wheat heads and $20 \mathrm{~g}$ of agar in 1 liter of water) for mycelial growth tests and in MBL (40 $\mathrm{g}$ of mung beans boiled in 1 liter water for 20 min and then filtered through cheesecloth) for sporulation analysis.

\section{Generation of gene deletion and complementation mutants.}

The double-joint PCR approach (Yu et al. 2004) was used to generate the gene replacement construct for each target gene. The resulting PCR products for each gene were transformed into protoplasts of the wild-type $\mathrm{PH}-1$ as described previously (Hou et al. 2002; Proctor et al. 1995a). Hygromycin B (Calbiochem) was added to a final concentration of $100 \mathrm{mg} / \mathrm{liter}$ for transformant selection. The putative targeted gene-deletion mutants were identified by PCR assays and were further confirmed by Southern hybridization assays. All the mutants generated in this study were preserved in $15 \%$ glycerol at $-80^{\circ} \mathrm{C}$.

The $F g R R D 1$ deletion mutant $\triangle$ FgRrd1 was complemented with the full $F g R R D 1$ gene. The DNA fragment of $F g R R D 1$ driven by the native promoter was inserted into pYF11 vector by using the yeast homologous recombination approach $(\mathrm{Ma}$ et al. 1987). The protoplast transformations of $\Delta$ FgRrd1 with the pYF11 recombination vectors were conducted as described above except that geneticin was used as a selection agent.
Using the same method, FgRrd2 deletion mutant was also complemented.

\section{Yeast complementation assays.}

Full-lengths cDNA of $F g R R D 1$ or $F g R R D 2$ were cloned into the pYES2 vector (Invitrogen Co.) and were transformed into the corresponding yeast mutant. Additionally, the wild-type strain BY4741 and a mutant transformed with an empty pYES2 vector were used as controls. For complementation assays, the yeast transformants were grown at $30^{\circ} \mathrm{C}$ on YPRG medium $(1 \%$ yeast extract, $2 \%$ bactopeptone, $2 \%$ galactose) supplemented with $0.05 \mu \mathrm{g}$ of rapamycin per milliliter. The experiments were repeated three times independently.

\section{Asexual and sexual development and growth tests.}

For conidiation assay, five mycelial plugs ( $5 \mathrm{~mm}$ in diameter) of each strain taken from the periphery of a 3-day-old colony were inoculated in a $50-\mathrm{ml}$ flask containing $20 \mathrm{ml}$ of MBL medium. The flasks were incubated at $25^{\circ} \mathrm{C}$ for 4 days in a shaker $(180 \mathrm{rpm})$. The amount of conidia in each flask was counted, using a hemacytometer. In addition, conidia of each strain were resuspended in $2 \%(\mathrm{wt} / \mathrm{vol})$ sucrose solutions and were incubated at $25^{\circ} \mathrm{C}$ for $6 \mathrm{~h}$, and then, conidial germination was examined under a Nikon ECLIPSE E100 microscope (Nikon Co.). The experiment was repeated three times independently. Conidial morphology was observed with a Leica7 TCS SP5 imaging system. Furthermore, septum and nucleus were examined after staining with CFW and 4',6-diamidino-2phenylindole, respectively, as described previously (Seong et al. 2008).

For sexual development assays, aerial hyphae of the 7-dayold culture of each strain on carrot agar were pressed down with $500 \mu \mathrm{l}$ of sterilized $0.3 \%$ Tween 20 . Perithecium formation was examined after 1 week of incubation at $25^{\circ} \mathrm{C}$, as described previously ( $\mathrm{Gu}$ et al. 2015).

For growth tests under various conditions, serial dilutions of conidial suspension of each strain were spotted on PDA amended with an antifungal compound, i.e., fludioxonil, carbendazim, tebuconazole, or phenamacril, and on MM supplemented with oxidative stress generator paraquat, cell-wall stress agent Congo red, $\mathrm{CFW}$, or SDS. After incubation at $25^{\circ} \mathrm{C}$ for 2 days, each plate was examined. Each experiment was repeated three times independently.

\section{Determination of DON production and virulence.}

A 50-g aliquot of healthy wheat kernels was sterilized and inoculated with five mycelial plugs of each strain. A negative control was prepared with the wheat kernels inoculated with five agar plugs. After incubation at $25^{\circ} \mathrm{C}$ for 20 days, the extraction and measurement of DON and ergosterol were performed as previously described, using a high-pressure liquid chromatography system Waters 1525 (Jiang et al. 2012; Mirocha et al. 1998). The experiment was repeated three times, and data were analyzed using analysis of variance (SAS version 8.0; SAS Institute). Virulence determination was performed with single floret injection method as previously described (Wu et al. 2005). The experiment was repeated four times.

\section{Histochemical analysis of lipid droplets.}

Lipid droplets were visualized by staining with Nile Red or Bodipy. Briefly, hyphae were incubated in the Nile Red staining solution (per milliliter, $20 \mathrm{mg}$ of polyvinylpyrrolidone and $2.5 \mathrm{mg}$ of Nile Red Oxazone [Sigma-Aldrich]) in $50 \mathrm{mM}$ Trismaleate buffer ( $\mathrm{pH}$ 7.5) for a few seconds, and then, lipid droplets were observed under a microscope with an episcopic fluorescence attachment. For Bodipy staining, hyphae were stained for $10 \mathrm{~min}$ in the dark with $1 \mu \mathrm{M}$ of Bodipy $493 / 503$ 
(ThermoFisher Scientific) dissolved in $0.1 \times$ phosphate buffered saline (PBS). After washing twice with PBS, lipid droplets were detected with the Zeiss LSM780 confocal microscope (Carl Zeiss AG). Each experiment was repeated three times.

\section{RNA extraction and qRT-PCR.}

Total RNA was extracted from mycelia of each sample, using the TaKaRa RNAiso reagent (TaKaRa Biotechnology Co.), and $10 \mathrm{mg}$ of each RNA sample was used for reverse transcription with a RevertAid H Minus First Strand cDNA synthesis kit employing the oligo(dT) $)_{18}$ primer (Fermentas Life Sciences). The expression levels of each gene under different culture conditions were determined by qRT-PCR. qPCR amplifications were performed with the DNA Engine Opticons 4 system (MJ Research), using SYBR Green I fluorescent dye detection. For each sample, PCR amplification for the quantification of the expression of the $F g A C T I N$ gene was performed as a reference. The experiment was repeated three times independently.

\section{Western blot analysis.}

Six mycelial plugs were inoculated into $150 \mathrm{ml}$ of potato dextrose broth (PDB) and was incubated at $25^{\circ} \mathrm{C}$ with agitation (200 rpm) for $36 \mathrm{~h}$. Mycelia were harvested and washed with deionized water. About 150 to $200 \mathrm{mg}$ of finely ground mycelia were resuspended in $1 \mathrm{ml}$ of extraction buffer $(50 \mathrm{mM}$ Tris$\mathrm{HCl}$, pH7.5, $100 \mathrm{mM} \mathrm{NaCl}, 5 \mathrm{mM}$ EDTA, 1\% Triton X-100, $2 \mathrm{mM}$ phenylmethylsulfonyl fluoride [PMSF]) and $10 \mu \mathrm{l}$ of protease inhibitor cocktail (Sangon). After homogenization with a vortex shaker, the lysate was centrifuged at full speed in a microcentrifuge for $20 \mathrm{~min}$ at $4^{\circ} \mathrm{C}$. About $50 \mu \mathrm{l}$ of supernatant was mixed with an equal volume of $2 \times$ loading buffer and was boiled for $5 \mathrm{~min}$. Then, $10 \mu \mathrm{l}$ of each sample was loaded onto SDS-polyacrylamide gel electrophoresis (PAGE) gels. Proteins separated on SDS-PAGE gels were transferred onto a polyvinylidene fluoride membrane with a Bio-Rad electroblotting apparatus.

The polyclonal anti-Flag A9044 (Sigma) and monoclonal anti-GFP ab32146 (Abcam) antibodies were used at a 1:5,000 to $1: 10,000$ dilution for immunoblot analyses. FgPp2A and methylated FgPp2A were detected using anti-Pp2A (Abcam) and antimethylation Leu309 of FgPp2A (anti-Pp2A-Me) (Abcam) antibodies, respectively. Monoclonal antibody anti-phosphoCTD (Cell Signaling Technology Inc.) was used for RNA polymerase II detection. Phosphorylated FgHog1 and FgHog1 were detected by the antibody against dually phosphorylated p38 (Thr180/Tyr182) (Cell Signaling Technology Inc.) and an anti-Hog1 antibody (Santa Cruz Biotechnology), respectively. The phosphorylated FgMgv1 and FgGpmk1 were detected with phospho-p44/42 MAPK antibody (Cell Signaling Technology Inc.). The total $\mathrm{FgMgv} 1$ and $\mathrm{FgGpmk} 1$ were detected using anti-Mpk1 (Santa Cruz Biotechnology) and p44/42 MAPK antibodies (Cell Signaling Technology Inc.), respectively. The samples were also detected with monoclonal anti-GAPDH antibody EM1101 (Hangzhou HuaAn Biotechnology Co., Ltd.) as a reference. Incubation with a secondary antibody and chemiluminescent detection were performed as described previously (Yang et al. 2012). The experiment was conducted three times independently.

\section{Co-IP assay.}

FgRrd1 and FgRrd2 were separately amplified and cloned into pYF11 (Zhou et al. 2011a), by the yeast gap repair approach (Bruno et al. 2004), to generate the GFP fusion con-

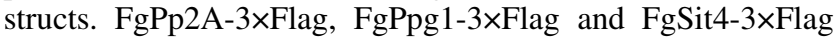
were cloned into the PDL2 vector according to the previous study (Yu et al. 2014; Zhou et al. 2011a). The resulting fusion constructs were verified by DNA sequencing and were transformed in pairs into the wild-type PH-1. Transformants expressing the fusion constructs were confirmed by Western blot analysis. For Co-IP assays, total proteins were isolated and incubated with the anti-GFP (ChromoTek) or anti-Flag (Abmart) agarose according to the manufacturer's instructions. After incubation at $4{ }^{\circ} \mathrm{C}$ overnight, the agarose beads were washed three times with $500 \mu \mathrm{l}$ of TBS $(20 \mathrm{mM}$ Tris- $\mathrm{HCl}, 500 \mathrm{mM} \mathrm{NaCl}, \mathrm{pH}$ 7.5). Proteins eluted from agarose were analyzed by Western blot detection with monoclonal anti-GFP ab32146 (Abcam), polyclonal anti-Flag A9044 (Sigma) or monoclonal anti-phosphoCTD antibodies (Cell Signaling Technology) for the detection of RNA polymerase II. The total protein samples were also detected with anti-GAPDH antibody EM1101 (Hangzhou HuaAn Biotechnology co., Ltd.) as a reference.

\section{Y2H assays.}

To construct plasmids for $\mathrm{Y} 2 \mathrm{H}$ analyses, the coding sequence of each tested gene was amplified from the cDNA of PH-1. The cDNA of each gene was inserted into the yeast GAL4 binding domain vector pGBKT7 and GAL4 activation domain vector pGADT7 (Clontech), respectively. The pairs of Y2H plasmids were cotransformed into $S$. cerevisiae AH109, following the LiAc/ SS-DNA/polyethylene glycol transformation protocol (Schiestl and Gietz 1989). In addition, a pair of plasmids, pGBKT7-53 and pGADT7, served as a positive control. A pair of plasmids, pGBKT7-Lam and pGADT7, was used as a negative control. Transformants were grown at $30^{\circ} \mathrm{C}$ for 3 days on synthetic defined (SD) medium lacking Leu and Trp and were then transferred to SD stripped of His, Leu and Trp and containing $5 \mathrm{mM} 3$-aminotriazole (3-AT) to assess binding activity (Jiang et al. 2011). Three independent experiments were performed to confirm $\mathrm{Y} 2 \mathrm{H}$ assay results. The transformants were also assayed for $\beta$-galactosidase activity following the previously published protocol (Zhou et al. 2011b). Three independent experiments were performed.

\section{ChIP and qRT analysis.}

ChIP was performed as previously described (Bernreiter et al. 2007; Chujo and Scott 2014; Reyes-Dominguez et al. 2012; Saleh et al. 2008) with additional modifications. Briefly, fresh mycelia were harvested and cross-linked with $1 \%$ formaldehyde for $15 \mathrm{~min}$. Fixation was stopped with $125 \mathrm{mM}$ glycine, and the cultures were then filtered and were immediately frozen in liquid nitrogen. The frozen cultures were ground with liquid nitrogen and were resuspended with $10 \mathrm{ml}$ of nuclear extraction buffer I (0.4 M sucrose, $10 \mathrm{mM}$ Tris- $\mathrm{HCl}, \mathrm{pH} 8.0$, $10 \mathrm{mM} \mathrm{MgCl} 2,5 \mathrm{mM} \beta$-mercaptoethanol, $0.1 \mathrm{mM}$ PMSF, $1 \times$ protease inhibitor). After centrifugation at $4,000 \times g$ at $4^{\circ} \mathrm{C}$ for $20 \mathrm{~min}$, the pellets were resuspended in $4 \mathrm{ml}$ of nuclear extraction buffer II (1.7 M sucrose, $10 \mathrm{mM}$ Tris- $\mathrm{HCl}, \mathrm{pH} 8.0$, $10 \mathrm{mM} \mathrm{MgCl} 2,1 \%$ Triton X-100, $5 \mathrm{mM} \beta$-mercaptoethanol, and $1 \times$ protease inhibitor) and were subsequently centrifuged at $13,000 \times g$ at $4^{\circ} \mathrm{C}$ for $15 \mathrm{~min}$. The resulting pellets were resuspended in $300 \mu \mathrm{l}$ of nuclear lysis buffer $(50 \mathrm{mM}$ Tris- $\mathrm{HCl}$, $\mathrm{pH}$ 8.0, $10 \mathrm{mM}$ EDTA, $1 \%$ SDS, and $1 \times$ protease inhibitor) and were then sonicated with two pulses of $30 \mathrm{~s}$ and $1 \mathrm{~min}$ of resting. After centrifugation, the supernatant was transformed into a clear tube and were diluted with $10 \times \mathrm{ChIP}$ dilution buffer (1.1\% Triton X-100, $1.2 \mathrm{mM}$ EDTA, $16.7 \mathrm{mM}$ Tris-HCl, $\mathrm{pH}$ 8.0 , and $167 \mathrm{mM} \mathrm{NaCl}$ ). Subsequently, immunoprecipitation was performed using monoclonal antibody anti-phospho-CTD (Cell Signaling Technology) together with the protein A-agarose (Santa Cruz Biotechnology), respectively. After washing, eluting, reverse cross-linking, and removing all proteins, the pellets were resuspended with $50 \mu \mathrm{l}$ of distilled water. The resulting cross-linked immunoprecipitated DNA was extracted by phenol/chloroform. Finally, qPCR amplifications were conducted as described above. Relative enrichment values were calculated by dividing the 
immunoprecipitated DNA by the input DNA. The experiments were repeated three times independently.

\section{Assay of PP2A activity.}

Phosphatase activity was measured using a calcineurin assay kit (Biomol) (Erental et al. 2007) and the detail protocols referenced to one previous study (Zhong et al. 2014). Total proteins were extracted from each strain grown in PDB for $36 \mathrm{~h}$, as described above. PP2A activity was measured as the dephosphorylation rate of a synthetic phosphopeptide substrate (RII peptide) in the presence or absence of $10 \mathrm{mM}$ EGTA buffer. The amount of liberated $\mathrm{PO}^{3}$ was determined colorimetrically. Results were normalized based on the protein concentration in each sample. The soluble protein content of the supernatant was determined using a dye-binding assay (Bradford 1976).

\section{LITERATURE CITED}

Albataineh, M. T., Lazzell, A., Lopez-Ribot, J. L., and Kadosh, D. 2014. Ppg1, a PP2A-type protein phosphatase, controls filament extension and virulence in Candida albicans. Eukaryot. Cell 13:1538-1547.

Bernal, M., Sanchez-Romero, M. A., Salas-Pino, S., and Daga, R. R. 2012. Regulation of fission yeast morphogenesis by PP2A activator pta2. PLoS One 7:e32823.

Bernreiter, A., Ramon, A., Fernández-Martínez, J., Berger, H., AraújoBazan, L., Espeso, E. A., Pachlinger, R., Gallmetzer, A., Anderl, I. Scazzocchio, C., and Strauss, J. 2007. Nuclear export of the transcription factor NirA is a regulatory checkpoint for nitrate induction in Aspergillus nidulans. Mol. Cell. Biol. 27:791-802.

Bradford, M. M. 1976. A rapid and sensitive method for the quantitation of microgram quantities of protein utilizing the principle of protein-dye binding. Anal. Biochem. 72:248-254.

Bruno, K. S., Tenjo, F., Li, L., Hamer, J. E., and Xu, J. R. 2004. Cellular localization and role of kinase activity of PMK1 in Magnaporthe grisea. Eukaryot. Cell 3:1525-1532.

Cayla, X., Van Hoof, C., Bosch, M., Waelkens, E., Vandekerckhove, J., Peeters, B., Merlevede, W., and Goris, J. 1994. Molecular cloning, expression, and characterization of PTPA, a protein that activates the tyrosyl phosphatase activity of protein phosphatase 2A. J. Biol. Chem. 269:15668-15675.

Chujo, T., and Scott, B. 2014. Histone H3K9 and H3K27 methylation regulates fungal alkaloid biosynthesis in a fungal endophyte-plant symbiosis. Mol. Microbiol. 92:413-434.

Desai, A., and Mitchison, T. J. 1997. Microtubule polymerization dynamics. Annu. Rev. Cell Dev. Biol. 13:83-117.

Desjardins, A. E., Proctor, R. H., Bai, G. H., Mccormick, S. P., Shaner, G., Buechley, G., and Hohn, T. M. 1996. Reduced virulence of trichothecenenonproducing mutants of Gibberella zeae in wheat field tests. Mol. PlantMicrobe Interact. 9:775-781.

Douville, J., David, J., Fortier, P. K., and Ramotar, D. 2004. The yeast phosphotyrosyl phosphatase activator protein, yPtpa1/Rrd1, interacts with Sit4 phosphatase to mediate resistance to 4-nitroquinoline-1-oxide and UVA. Curr. Genet. 46:72-81.

Du, Y., Shi, Y., Yang, J., Chen, X., Xue, M., Zhou, W., and Peng, Y. L. 2013. A serine/threonine-protein phosphatase PP2A catalytic subunit is essential for asexual development and plant infection in Magnaporthe oryzae. Curr. Genet. 59:33-41.

Egan, J. D., García-Pedrajas, M. D., Andrews, D. L., and Gold, S. E. 2009. Calcineurin is an antagonist to PKA protein phosphorylation required for postmating filamentation and virulence, while PP2A is required for viability in Ustilago maydis. Mol. Plant-Microbe Interact. 22:1293-1301.

Erental, A., Harel, A., and Yarden, O. 2007. Type 2A phosphoprotein phosphatase is required for asexual development and pathogenesis of Sclerotinia sclerotiorum. Mol. Plant-Microbe Interact. 20:944-954.

Fellner, T., Lackner, D. H., Hombauer, H., Piribauer, P., Mudrak, I., Zaragoza, K., Juno, C., and Ogris, E. 2003. A novel and essential mechanism determining specificity and activity of protein phosphatase 2A (PP2A) in vivo. Genes Dev. 17:2138-2150.

Flández, M., Cosano, I. C., Nombela, C., Martín, H., and Molina, M. 2004. Reciprocal regulation between Slt2 MAPK and isoforms of Msg5 dualspecificity protein phosphatase modulates the yeast cell integrity pathway. J. Biol. Chem. 279:11027-11034.

Gu, Q., Zhang, C., Liu, X., and Ma, Z. 2015. A transcription factor FgSte12 is required for pathogenicity in Fusarium graminearum. Mol. Plant Pathol. 16:1-13.
Guo, F., Stanevich, V., Wlodarchak, N., Sengupta, R., Jiang, L., Satyshur, K. A., and Xing, Y. 2014. Structural basis of PP2A activation by PTPA, an ATP-dependent activation chaperone. Cell Res. 24:190-203.

Hombauer, H., Weismann, D., Mudrak, I., Stanzel, C., Fellner, T., Lackner, D. H., and Ogris, E. 2007. Generation of active protein phosphatase 2A is coupled to holoenzyme assembly. PLoS Biol. 5:e155.

Hou, Z., Xue, C., Peng, Y., Katan, T., Kistler, H. C., and Xu, J. R. 2002. A mitogen-activated protein kinase gene (MGV1) in Fusarium graminearum is required for female fertility, heterokaryon formation, and plant infection. Mol. Plant-Microbe Interact. 15:1119-1127.

Janssens, V., Van Hoof, C., De Baere, I., Merlevede, W., and Goris, J. 1999. Functional analysis of the promoter region of the human phosphotyrosine phosphatase activator gene: Yin Yang 1 is essential for core promoter activity. Biochem. J. 344:755-763

Janssens, V., Van Hoof, C., De Baere, I., Merlevede, W., and Goris, J. 2000. The phosphotyrosyl phosphatase activator gene is a novel p53 target gene. J. Biol. Chem. 275:20488-20495.

Jiang, J., Yun, Y., Liu, Y., and Ma, Z. 2012. FgVELB is associated with vegetative differentiation, secondary metabolism and virulence in Fusarium graminearum. Fungal Genet. Biol. 49:653-662.

Jiang, J., Yun, Y., Yang, Q., Shim, W. B., Wang, Z., and Ma, Z. 2011. A type 2C protein phosphatase $\mathrm{FgPtc} 3$ is involved in cell wall integrity, lipid metabolism, and virulence in Fusarium graminearum. PLoS One 6:e25311.

Jordens, J., Janssens, V., Longin, S., Stevens, I., Martens, E., Bultynck, G., Engelborghs, Y., Lescrinier, E., Waelkens, E., Goris, J., and Van Hoof, C. 2006. The protein phosphatase $2 \mathrm{~A}$ phosphatase activator is a novel peptidyl-prolyl cis/trans-isomerase. J. Biol. Chem. 281:6349-6357.

Jouvet, N., Poschmann, J., Douville, J., Bulet, L., and Ramotar, D. 2010. Rrd1 isomerizes RNA polymerase II in response to rapamycin. BMC Mol. Biol. 11:92.

Kaeberlein, M., Andalis, A. A., Liszt, G. B., Fink, G. R., and Guarente, L. 2004. Saccharomyces cerevisiae SSD1-V confers longevity by a Sir2pindependent mechanism. Genetics 166:1661-1672.

Kojima, K., Kikuchi, T., Takano, Y., Oshiro, E., and Okuno, T. 2002. The mitogen-activated protein kinase gene MAF1 is essential for the early differentiation phase of appressorium formation in Colletotrichum lagenarium. Mol. Plant-Microbe Interact. 15:1268-1276.

Leulliot, N., Vicentini, G., Jordens, J., Quevillon-Cheruel, S., Schiltz, M. Barford, D., van Tilbeurgh, H., and Goris, J. 2006. Crystal structure of the PP2A phosphatase activator: Implications for its PP2A-specific PPIase activity. Mol. Cell 23:413-424.

Longin, S., Jordens, J., Martens, E., Stevens, I., Janssens, V., Rondelez, E., De Baere, I., Derua, R., Waelkens, E., Goris, J., and Van Hoof, C. 2004 An inactive protein phosphatase $2 \mathrm{~A}$ population is associated with methylesterase and can be re-activated by the phosphotyrosyl phosphatase activator. Biochem. J. 380:111-119.

Luo, Y., Nie, Y. J., Shi, H. R., Ni, Z. F., Wang, Q., Wang, J. Z., and Liu, G. P. 2013. PTPA activates protein phosphatase-2A through reducing its phosphorylation at tyrosine-307 with upregulation of protein tyrosine phosphatase 1B. Biochim. Biophys. Acta 1833:1235-1243.

Ma, H., Kunes, S., Schatz, P. J., and Botstein, D. 1987. Plasmid construction by homologous recombination in yeast. Gene 58:201-216.

McMullen, M., Jones, R., and Gallenberg, D. 1997. Scab of wheat and barley: A re-emerging disease of devastating impact. Plant Dis. 81:1340-1348.

Min, K., Shin, Y., Son, H., Lee, J., Kim, J. C., Choi, G. J., and Lee, Y. W. 2012. Functional analyses of the nitrogen regulatory gene areA in Gibberella zeae. FEMS Microbiol. Lett. 334:66-73.

Mirocha, C. J., Kolaczkowski, E., Xie, W., Yu, H., and Jelen, H. 1998. Analysis of deoxynivalenol and its derivatives (batch and single kernel) using gas chromatography/mass spectrometry. J. Agric. Food Chem. 46:1414-1418.

Mumby, M. 2007. PP2A: Unveiling a reluctant tumor suppressor. Cell 130: 21-24.

Pestka, J. J., and Smolinski, A. T. 2005. Deoxynivalenol: Toxicology and potential effects on humans. J. Toxicol. Environ. Health B Crit. Rev. 8:39-69.

Poschmann, J., Drouin, S., Jacques, P. E., El Fadili, K., Newmarch, M., Robert, F., and Ramotar, D. 2011. The peptidyl prolyl isomerase Rrd1 regulates the elongation of RNA polymerase II during transcriptional stresses. PLoS One 6:e23159.

Proctor, R. H., Hohn, T. M., and McCormick, S. P. 1995a. Reduced virulence of Gibberella zeae caused by disruption of a trichothecene toxin biosynthetic gene. Mol. Plant-Microbe Interact. 8:593-601.

Proctor, R. H., Hohn, T. M., McCormick, S. P., and Desjardins, A. E. 1995b. Tri6 encodes an unusual zinc finger protein involved in regulation of trichothecene biosynthesis in Fusarium sporotrichioides. Appl. Environ. Microbiol. 61:1923-1930.

Qi, W., Kwon, C., and Trail, F. 2006. Microarray analysis of transcript accumulation during perithecium development in the filamentous fungus Gibberella zeae (anamorph Fusarium graminearum). Mol. Genet. Genomics 276:87-100. 
Rempola, B., Kaniak, A., Migdalski, A., Rytka, J., Slonimski, P. P., and di Rago, J. P. 2000. Functional analysis of RRD1 (YIL153w) and RRD2 (YPL152w), which encode two putative activators of the phosphotyrosyl phosphatase activity of PP2A in Saccharomyces cerevisiae. Mol. Gen. Genet. 262:1081-1092.

Reyes-Dominguez, Y., Boedi, S., Sulyok, M., Wiesenberger, G., Stoppacher, N., Krska, R., and Strauss, J. 2012. Heterochromatin influences the secondary metabolite profile in the plant pathogen Fusarium graminearum. Fungal Genet. Biol. 49:39-47.

Saleh, A., Alvarez-Venegas, R., and Avramova, Z. 2008. An efficient chromatin immunoprecipitation (ChIP) protocol for studying histone modifications in Arabidopsis plants. Nat. Protoc. 3:1018-1025.

Schiestl, R. H., and Gietz, R. D. 1989. High efficiency transformation of intact yeast cells using single stranded nucleic acids as a carrier. Curr. Genet. 16:339-346.

Sen, R., Malik, S., Frankland-Searby, S., Uprety, B., Lahudkar, S., and Bhaumik, S. R. 2014. Rrd1p, an RNA polymerase II-specific prolyl isomerase and activator of phosphoprotein phosphatase, promotes transcription independently of rapamycin response. Nucleic Acids Res. 42:9892-9907.

Sents, W., Ivanova, E., Lambrecht, C., Haesen, D., and Janssens, V. 2013. The biogenesis of active protein phosphatase $2 \mathrm{~A}$ holoenzymes: A tightly regulated process creating phosphatase specificity. FEBS J. 280:644-661.

Seong, K. Y., Zhao, X., Xu, J. R., Güldener, U., and Kistler, H. C. 2008. Conidial germination in the filamentous fungus Fusarium graminearum. Fungal Genet. Biol. 45:389-399.

Shi, Y. 2009. Serine/threonine phosphatases: Mechanism through structure. Cell 139:468-484.

Shin, J. H., Kim, J. E., Malapi-Wight, M., Choi, Y. E., Shaw, B. D., and Shim, W. B. 2013. Protein phosphatase 2 A regulatory subunits perform distinct functional roles in the maize pathogen Fusarium verticillioides. Mol. Plant Pathol. 14:518-529.

Son, H., Seo, Y. S., Min, K., Park, A. R., Lee, J., Jin, J. M., Lin, Y., Cao, P., Hong, S. Y., Kim, E. K., Lee, S. H., Cho, A., Lee, S., Kim, M. G., Kim, Y., Kim, J. E., Kim, J. C., Choi, G. J., Yun, S. H., Lim, J. Y., Kim, M., Lee, Y. H., Choi, Y. D., and Lee, Y. W. 2011. A phenome-based functional analysis of transcription factors in the cereal head blight fungus, Fusarium graminearum. PLoS Pathog. 7:e1002310.

Stanevich, V., Jiang, L., Satyshur, K. A., Li, Y., Jeffrey, P. D., Li, Z., Menden, P., Semmelhack, M. F., and Xing, Y. 2011. The structural basis for tight control of PP2A methylation and function by LCMT-1. Mol. Cell 41:331-342.

Starkey, D. E., Ward, T. J., Aoki, T., Gale, L. R., Kistler, H. C., Geiser, D. M., Suga, H., Tóth, B., Varga, J., and O’Donnell, K. 2007. Global molecular surveillance reveals novel Fusarium head blight species and trichothecene toxin diversity. Fungal Genet. Biol. 44:1191-1204.

Trail, F. 2009. For blighted waves of grain: Fusarium graminearum in the postgenomics era. Plant Physiol. 149:103-110.

Van Hoof, C., Janssens, V., De Baere, I., de Winde, J. H., Winderickx, J., Dumortier, F., Thevelein, J. M., Merlevede, W., and Goris, J. 2000. The Saccharomyces cerevisiae homologue YPA1 of the mammalian phosphotyrosyl phosphatase activator of protein phosphatase 2A controls progression through the G1 phase of the yeast cell cycle. J. Mol. Biol. 302:103-119.

Van Hoof, C., Janssens, V., De Baere, I., Stark, M. J. R., de Winde, J. H., Winderickx, J., Thevelein, J. M., Merlevede, W., and Goris, J. 2001. The
Saccharomyces cerevisiae phosphotyrosyl phosphatase activator proteins are required for a subset of the functions disrupted by protein phosphatase 2A mutations. Exp. Cell Res. 264:372-387.

Van Hoof, C., Janssens, V., Dinishiotu, A., Merlevede, W., and Goris, J. 1998. Functional analysis of conserved domains in the phosphotyrosyl phosphatase activator. Molecular cloning of the homologues from Drosophila melanogaster and Saccharomyces cerevisiae. Biochemistry 37:12899-12908.

Van Hoof, C., Martens, E., Longin, S., Jordens, J., Stevens, I., Janssens, V., and Goris, J. 2005. Specific interactions of PP2A and PP2A-like phosphatases with the yeast PTPA homologues, Ypa1 and Ypa2. Biochem. J. 386:93-102.

Wang, C., Zhang, S., Hou, R., Zhao, Z., Zheng, Q., Xu, Q., Zheng, D., Wang, G., Liu, H., Gao, X., Ma, J.-W., Kistler, H. C., Kang, Z., and Xu, J.-R. 2011. Functional analysis of the kinome of the wheat scab fungus Fusarium graminearum. PLoS Pathog. 7:e1002460.

Westermarck, J., and Hahn, W. C. 2008. Multiple pathways regulated by the tumor suppressor PP2A in transformation. Trends Mol. Med. 14: $152-160$.

Wu, A.-B., Li, H.-P., Zhao, C.-S., and Liao, Y.-C. 2005. Comparative pathogenicity of Fusarium graminearum isolates from China revealed by wheat coleoptile and floret inoculations. Mycopathologia 160:75-83.

Xu, Y., Xing, Y., Chen, Y., Chao, Y., Lin, Z., Fan, E., Yu, J. W., Strack, S., Jeffrey, P. D., and Shi, Y. 2006. Structure of the protein phosphatase 2A holoenzyme. Cell 127:1239-1251.

Yang, Q., Yan, L., Gu, Q., and Ma, Z. 2012. The mitogen-activated protein kinase kinase kinase $\mathrm{BcOs} 4$ is required for vegetative differentiation and pathogenicity in Botrytis cinerea. Appl. Microbiol. Biotechnol. 96: 481-492.

Yu, F., Gu, Q., Yun, Y., Yin, Y., Xu, J. R., Shim, W. B., and Ma, Z. 2014. The TOR signaling pathway regulates vegetative development and virulence in Fusarium graminearum. New Phytol. 203:219-232.

Yu, J. H., Hamari, Z., Han, K. H., Seo, J. A., Reyes-Domínguez, Y., and Scazzocchio, C. 2004. Double-joint PCR: A PCR-based molecular tool for gene manipulations in filamentous fungi. Fungal Genet. Biol. 41:973-981.

Zheng, Y., and Jiang, Y. 2005. The yeast phosphotyrosyl phosphatase activator is part of the Tap42-phosphatase complexes. Mol. Biol. Cell 16: 2119-2127.

Zhong, G. W., Jiang, P., Qiao, W. R., Zhang, Y. W., Wei, W. F., and Lu, L. 2014. Protein phosphatase 2A (PP2A) regulatory subunits ParA and PabA orchestrate septation and conidiation and are essential for PP2A activity in Aspergillus nidulans. Eukaryot. Cell 13:1494-1506.

Zhou, X., Li, G., and Xu, J. R. 2011a. Efficient approaches for generating GFP fusion and epitope-tagging constructs in filamentous fungi. Methods Mol. Biol. 722:199-212.

Zhou, X., Liu, W., Wang, C., Xu, Q., Wang, Y., Ding, S., and Xu, J. R. 2011b. A MADS-box transcription factor MoMcml is required for male fertility, microconidium production and virulence in Magnaporthe oryzae. Mol. Microbiol. 80:33-53.

\section{AUTHOR-RECOMMENDED INTERNET RESOURCES}

Fusarium genome database: http://fungi.ensembl.org/index.html

The National Center for Biotechnology Information website: http://www.ncbi.nlm.nih.gov 